\title{
An Extension of Ausubel's Auction for Heterogeneous Discrete Goods
}

\author{
Hakan İnal ${ }^{*}$ \\ Department of Economics, School of Business and \\ Center for Public Policy, L. Douglas Wilder School of Government and Public Affairs \\ Virginia Commonwealth University \\ hinal@vcu.edu
}

February 5, 2011

\begin{abstract}
Ausubel's dynamic private-values auction for heterogeneous discrete goods, Ausubel (2006), yields an efficient equilibrium outcome but it is designed for a limited class of environments. If bidders' values for bundles of goods are not integers, then the auction mechanism may not yield an efficient allocation without any information on bidders' values. In this paper, I extend Ausubel's auction for heterogeneous discrete goods to real-valued quasilinear utility functions. The mechanism I propose reaches a Walrasian equilibrium price vector in finite "steps" without any additional information on bidders' values. In the extension of Ausubel's auction, truthful bidding constitutes an efficient equilibrium.
\end{abstract}

*I would like to thank my advisors Ket Richter and Jan Werner for their continuous support, patience, and effort for my education. Their guidance has been enlightening. I would like to thank late Leo Hurwicz, and Andy McLennan for their contribution to my education. I benefited a lot from discussions with David Rahman and Itai Sher. I also would like to thank Pat Bajari, Tuba İnal, Ichiro Obara, Raj Singh, and the seminar participants at Central Michigan University, the University of Minnesota, the Virginia Commonwealth University, Whitman College, the Games 2008, and the Society for Economic Design 2008 Conference for their valuable comments. All errors are mine. 
JEL Category D44

Keywords: Auctions, Ausubel auction, heterogeneous goods, discrete goods, price adjustment, tâtonnement.

\section{Introduction}

Auctioning of multiple goods has become a rapidly developing part of the auction theory since the Federal Communications Commission began auctioning wireless communication bands in 1994. It is well-known that when many units of a good are to be auctioned, standard auction mechanisms are generally inefficient, i.e., they do not award goods to buyers who value them most. This inefficiency arises from buyers' tendency to shade their bids, the demand-reduction problem (see Krishna (2002), Ausubel and Cramton (2002), and Milgrom (2004)). Vickrey's sealed-bid auction, Vickrey (1961), is among the few exceptions immune to the demand-reduction problem. Even though the Vickrey auction is efficient, it is not widely used in practice. In the Vickrey auction, bidders are supposed to submit their whole demand curves to the auctioneer. So, if there are 10 objects for sale, each bidder must compute and submit his values for $2^{10}$ bundles of goods. Bidders prefer not to submit so much information about themselves. Ausubel (2006) introduced an elegant dynamic auction for divisible and discrete heterogeneous goods, and it is immune to the demand-reduction problem. However, Ausubel's auction for heterogeneous discrete goods is designed for a limited class of environments: Bidders' values for bundles are restricted to be integers, and the tâtonnement algorithm (price adjustment procedure, see Ausubel (2005)) uses integer property of utility functions. If bidders' values for bundles are not integers, without information on these values, the tâtonnement algorithm may not reach a Walrasian equilibrium price vector. The reason for this integer restriction is not explained in his paper.

One may argue that Ausubel's auction, which uses the grid of integer prices, with sufficiently fine grid of prices is a straightforward approximation of Ausubel's auction to real numbers resulting in prices close to equilibrium prices. The first problem with this argument is that the integer assumption has a significant role in the price adjustment procedure: during the ascending price adjustment procedure, if bidders have substitutes preferences and they sincerely report their demand sets, at each integer price vector reached, the auctioneer determines bidders' indirect utility functions 
correctly for all prices in the $K$-dimensional unit cube above that integer price vector (See Ausubel (2005), p.619). Using these correct indirect utility functions, the auctioneer computes the next integer price vector in the price adjustment. If the integer assumption is relaxed, the auctioneer may not be able to correctly determine the indirect utility functions in the $K$ dimensional cube above some price vector no matter how small the size of the grid. As a result, the price adjustment procedure may not follow the price path it is designed to because the auctioneer does not have the correct information about indirect utility functions. The second problem is about the allocation of goods: Ausubel's auction is designed to allocate goods if and only if an equilibrium price is reached, and the corresponding market clearing allocation is used. There is no straightforward way to choose an allocation if the procedure stops at a non-equilibrium price vector. Moreover, it is not possible to get arbitrarily close to an efficient allocation by making the grid finer because goods are discrete.

In this paper, I extend Ausubel's auction for heterogeneous discrete goods to real-valued quasilinear utility functions by introducing an analogous extension of the tâtonnement algorithm in Ausubel (2006). I show that the extended tâtonnement algorithm reaches a Walrasian equilibrium price vector in finite "steps" when bidders' values are real numbers. The extended Ausubel auction for heterogeneous discrete goods has an efficient equilibrium and yields Walrasian equilibrium prices when bidders' values for bundles are real numbers. Unlike the tâtonnement algorithm of Ausubel (2006), in the extended tâtonnement algorithm, the auctioneer does not need any information on the values bidders' utility functions take when these values are not integers.

In Ausubel's auction, Ausubel (2006), bidders submit their demands as prices are adjusted. A bidder is credited a unit of a good at the current price when the rest of the bidders lower their demand for this good. A unit of a good is debited from a bidder at the current price when the rest of the bidders increase their demand for this good. The auctioneer calculates the set of goods in excess demand and adjusts the prices accordingly. The auction ends whenever there is a market clearing allocation demanded by bidders at the current price. Bidders are assumed to have private values for goods (each bidder's values for goods depend only on his own type) and have utility functions quasilinear in money. In the case of divisible goods, bidders have concave utility functions whereas in the case of discrete goods, they have preferences satisfying the gross substitutes condition. The gross 
substitutes assumption basically requires that a bidder's demand for a set of goods to be nondecreasing if their prices remain the same while the rest of the prices do not decrease. This assumption guarantees the existence of a Walrasian equilibrium (see Gul and Stacchetti (1999)). When goods are divisible, the classical Walrasian tâtonnement is used to determine the path of prices. In the case of discrete goods, a tâtonnement algorithm (see Ausubel (2005)) is used. There are two analogous versions of the tâtonnement algorithm: the ascending algorithm and the descending algorithm. Ausubel (2005) shows that the ascending (descending) algorithm reaches a Walrasian equilibrium price vector in finite steps if the initial prices are sufficiently small (large). Ausubel (2006) converts this discrete-time price adjustment procedure to a continuous-time price adjustment procedure. He achieves that by linearly increasing (or decreasing) prices between two consecutive integervalued price vectors determined by the tâtonnement algorithm. Then, he uses this continuous-time price adjustment procedure to prove that sincere bidding by bidders comprise an efficient equilibrium and yields Walrasian equilibrium prices.

As Ausubel's auction does not require bidders to reveal all of their demands at all prices, it preserves privacy of bidders, and it is simpler than the Vickrey auction. Also, bidders are allowed to exercise their market power but strategic bidders behave as price-takers under Ausubel's nonlinear pricing rule, which solves the demand-reduction problem.

In the proposed extension of Ausubel's auction for discrete heterogeneous goods, the auction starts at an initial price vector $\boldsymbol{p}(0)$. Each bidder submits his report, a set of bundle of goods he demands at $\boldsymbol{p}(0)$. Bidders are allowed to respond to each other's reports at the same price $\boldsymbol{p}(0)$. Rounds of these bidders' reports continue as long as there is a bidder who wants to report a demand set. After these rounds are over, the auctioneer adjusts prices continuously according to the extended tâtonnement algorithm using the demand reports from the last round. Bidders may add a new bundle to or remove a bundle from their demand set during the price adjustment. They submit their reports whenever they add a new bundle to their demand sets as prices change. At any time $t \in[0, \infty)$, if there is a bidder who submits a new report at the current price $\boldsymbol{p}(t)$, then the price adjustment stops. Again, bidders are allowed to respond to these reports. After rounds of bidding are over, prices are adjusted continuously according to the extended tâtonnement algorithm using the demand reports from the last round. During the price adjustment process, for each bidder $i \in N$, if opponents of bidder $i$ lower 
their demand for a good, then the good is credited to bidder $i$ at price $\boldsymbol{p}(t)$. On the other hand, if opponents of bidder $i$ rise their demand for a good, then it is debited from bidder $i$ at price $\boldsymbol{p}(t)$. The auction ends at time $T \in[0, \infty)$ when there is a market clearing allocation of goods in these reports made at time $T$. Payment of each bidder is calculated by adding his credits and debits, and market clearing allocation of goods are made.

Milgrom and Strulovici (2009) in a recent paper also extended the Ausubel auction independently. They use a steepest-descent algorithm whereas the algorithm used in this paper is an analogous extension of the tâtonnement algorithm of Ausubel (2006). In the extended algorithm proposed here, unlike Milgrom and Strulovici (2009), bidders do not reveal their demands continuously. Bidders report their demand sets finitely many times, whenever they add a new bundle to their demand sets during the price adjustment process.

Section 2 gives the assumptions of the model. In Section 3, the extension of Ausubel's auction and the extended tâtonnement algorithm are explained. Section 4 explains how to identify paths of the extended ascending and descending algorithms, and shows that they reach Walrasian equilibrium price vectors in finite steps.

\section{The Model}

In this paper, I follow a notation very similar to the one in Ausubel (2005) so that the reader can easily follow one paper after the other. Let $\mathbb{Z}, \mathbb{Q}$, and $\mathbb{R}$ stand for the sets of integer, rational and real numbers respectively. There are finite number of goods, $K=\{1,2, \ldots, K\}$. There is a seller with supply $\boldsymbol{S}=\left(S^{k}\right)_{k \in K} \in \mathbb{Z}_{++}^{K}$ of discrete heterogeneous goods, and she wants to sell them to a finite group of bidders, $N=\{1,2, \ldots, N\}$. Consumption set of bidder $i$ is $X_{i}=\left\{\boldsymbol{x} \in \mathbb{Z}^{K}: 0 \leq x^{k} \leq \bar{x}_{i}^{k}\right.$ for all $\left.k \in K\right\}$ where $\left(\bar{x}_{i}^{k}\right)_{k \in K} \in \mathbb{Z}^{K}$, and it is bounded below and above. $\boldsymbol{x}_{\boldsymbol{i}}=\left(x_{i}^{k}\right)_{k \in K} \in X_{i}$ is a bundle bidder $i$ consumes.

The following assumptions are made for each bidder $i$ :

A.1 Private Values: Bidder $i$ 's utility function $u_{i}: X_{i} \times \mathbb{R} \rightarrow \mathbb{R}$ is a function of bundle $\boldsymbol{x}_{\boldsymbol{i}} \in X_{i}$ and money $t_{i} \in \mathbb{R}$ he consumes, and it does not depend on any information about other bidders.

A.2 Quasilinearity: $u_{i}(\cdot)$ is assumed to be quasilinear in money, i.e., there exists $U_{i}: X_{i} \rightarrow \mathbb{R}$ such that for each $x_{i} \in X_{i}$ and each $t_{i} \in \mathbb{R}$, 


$$
u_{i}\left(\boldsymbol{x}_{\boldsymbol{i}}, t_{i}\right)=U_{i}\left(\boldsymbol{x}_{\boldsymbol{i}}\right)+t_{i}
$$

where $U_{i}\left(\boldsymbol{x}_{\boldsymbol{i}}\right)$ is $i$ 's value for bundle $\boldsymbol{x}_{\boldsymbol{i}}$.

Initial wealth $m_{i} \in \mathbb{R}_{++}$of each bidder $i \in N$ is so large that his budget constraint does not bind for any bundle he demands at any price. Since $u_{i}\left(\boldsymbol{x}_{\boldsymbol{i}}, m_{i}-y_{i}\right)=U_{i}\left(\boldsymbol{x}_{\boldsymbol{i}}\right)+m_{i}-y_{i}$ where $y_{i}$ is the amount of expense he makes, $m_{i}$ will be dropped without loss of generality.

A.3 Strict Monotonicity: For all $\left(\boldsymbol{x}_{\boldsymbol{i}}^{\prime}, t_{i}^{\prime}\right)$ and $\left(\boldsymbol{x}_{\boldsymbol{i}}, t_{i}\right) \in X_{i} \times \mathbb{R}$ such that $\left(\boldsymbol{x}_{\boldsymbol{i}}^{\prime}, t_{i}^{\prime}\right) \supsetneqq\left(\boldsymbol{x}_{\boldsymbol{i}}, t_{i}\right)$,

$$
u_{i}\left(\boldsymbol{x}_{\boldsymbol{i}}^{\prime}, t_{i}^{\prime}\right)>u_{i}\left(\boldsymbol{x}_{\boldsymbol{i}}, t_{i}\right)
$$

Bidder $i$ 's indirect utility function at price vector $\boldsymbol{p}=\left(p^{k}\right)_{k \in K} \in \mathbb{R}_{+}^{K}$ is

$$
V_{i}(\boldsymbol{p})=\max _{\boldsymbol{x}_{\boldsymbol{i}} \in X_{i}}\left\{U_{i}\left(\boldsymbol{x}_{\boldsymbol{i}}\right)-\boldsymbol{p} \cdot \boldsymbol{x}_{\boldsymbol{i}}\right\}
$$

and his demand correspondence (demand set) at price vector $\boldsymbol{p} \in \mathbb{R}_{+}^{K}$ is

$$
Q_{i}(\boldsymbol{p})=\underset{\boldsymbol{x}_{\boldsymbol{i}} \in X_{i}}{\arg \max }\left\{U_{i}\left(\boldsymbol{x}_{\boldsymbol{i}}\right)-\boldsymbol{p} \cdot \boldsymbol{x}_{\boldsymbol{i}}\right\} .
$$

A Walrasian equilibrium is $\left(\boldsymbol{p}^{*}, \boldsymbol{x}^{*}\right)$ where $\boldsymbol{p}^{*}$ is equilibrium price vector and $\boldsymbol{x}^{*}=\left(\boldsymbol{x}_{\boldsymbol{i}}\right)_{i \in N}$ is equilibrium allocation, such that for each bidder $i \in N$ $\boldsymbol{x}_{\boldsymbol{i}}^{*} \in Q_{i}\left(\boldsymbol{p}^{*}\right)$, and $\sum_{i \in N} \boldsymbol{x}_{\boldsymbol{i}}^{*}=\boldsymbol{S}$. Existence of Walrasian equilibrium heavily relies on the gross substitutes assumption. Gul and Stacchetti (1999) shows that the gross substitutes assumption is sufficient and "almost necessary" for the existence of the Walrasian equilibrium. In the gross substitutes assumption below, each good is assumed to be available in unit supply. The auction mechanism proposed in this paper, like Ausubel's auction Ausubel (2006), allows multiple units of each good without loss of generality (see Bikhchandani and Mamer (1997)). For more on the gross substitutes assumption see Milgrom and Strulovici (2009): they make an extensive analysis of the gross substitutes assumption and its relation to equilibrium.

A.4 Gross Substitutes: For all price vectors $\boldsymbol{p}$ and $\boldsymbol{p}^{\prime} \in \mathbb{R}_{+}^{K}$ such that $\boldsymbol{p}^{\prime} \geq \boldsymbol{p}$, if demand $Q_{i}(\cdot)$ is single-valued both at $\boldsymbol{p}$ and at $\boldsymbol{p}^{\prime}, \boldsymbol{x}_{\boldsymbol{i}} \in Q_{i}(\boldsymbol{p})$, and $\boldsymbol{x}_{\boldsymbol{i}}^{\prime} \in Q_{i}\left(\boldsymbol{p}^{\prime}\right)$, then $x_{i}^{\prime k} \geq x_{i}^{k}$ for each $k \in K$ such that $p^{\prime k}=p^{k}$.

In Ausubel's auction, Ausubel (2006), bidders' values for bundles are assumed to be integer. The tâtonnement algorithm in Ausubel (2006) is designed to use this property of utility functions to move on the grid of integer price vectors, and to reach an integer-valued Walrasian equilibrium 
price vector. If the integer assumption on values is relaxed, more information on bidders' values is needed to reach a Walrasian equilibrium price vector. There is no straightforward way of modifying the tâtonnement algorithm so that it will reach a Walrasian equilibrium. If bidders' values for bundles are not integer, then there may not exist an integer-valued Walrasian equilibrium price vector, and the price adjustment procedure may not yield an efficient allocation when it ends. As Lemma 1 below shows, relaxing the integer-values restriction enriches the class of preferences of bidders.

A preference relation $\mathcal{R}$ defined on $X \times \mathbb{R}$ is representable if there exists a utility function $u: X \times \mathbb{R} \rightarrow \mathbb{R}$ such that for each $\boldsymbol{x}, \boldsymbol{y} \in X \times \mathbb{R}$,

$$
\boldsymbol{x} \mathcal{R} \boldsymbol{y} \text { if and only if } u(\boldsymbol{x}) \geq u(\boldsymbol{y}) .
$$

A preference relation $\mathcal{R}$ is said to be in $\mathcal{R}_{D}$ if and only if there exists a quasilinear utility function $u: X \times \mathbb{R} \rightarrow \mathbb{R}$ representing $\mathcal{R}$ such that $u(\cdot, t)=U(\cdot)+t$ where $U: X \rightarrow D$.

Lemma 1 shows that sets $\mathcal{R}_{\mathbb{Z}}$, and $\mathcal{R}_{\mathbb{R}}$ are different.

Lemma 1. $\mathcal{R}_{\mathbb{Z}} \subsetneq \mathcal{R}_{\mathbb{R}}$.

Proof. $\mathcal{R}_{\mathbb{Z}} \subset \mathcal{R}_{\mathbb{R}}$ is trivial. I will show that there exists $\mathcal{R} \in \mathcal{R}_{\mathbb{R}}$ such that $\mathcal{R} \notin \mathcal{R}_{\mathbb{Z}}$. Let $\mathcal{R} \in \mathcal{R}_{\mathbb{R}}$ be such that there exist $\boldsymbol{x}, \boldsymbol{y} \in X$ and $\bar{t} \in \mathbb{R} \backslash \mathbb{Z}$ such that

$$
(\boldsymbol{x}, \bar{t}) \underset{\mathcal{R}}{\sim}(\boldsymbol{y}, 0) .
$$

By the definition of $\mathcal{R}_{\mathbb{R}}$, there exists $u(\cdot, t)=U(\cdot)+t$ representing $\mathcal{R}$, where $U: X \rightarrow \mathbb{R}$. Equation 1 implies that

$$
u(\boldsymbol{x}, \bar{t})=u(\boldsymbol{y}, 0) .
$$

Therefore,

$$
\bar{t}=U(\boldsymbol{y})-U(\boldsymbol{x}) \in \mathbb{R} \backslash \mathbb{Z} .
$$

Now, assume on the contrary that, there exists $\widetilde{u}(\cdot, t)=\widetilde{U}(\cdot)+t$ representing $\mathcal{R}$ such that

$$
\widetilde{U}: X \rightarrow \mathbb{Z} .
$$

Equation 1 implies that $\widetilde{u}(\boldsymbol{x}, \bar{t})=\widetilde{u}(\boldsymbol{y}, 0)$. So, $\bar{t}=\widetilde{U}(\boldsymbol{y})-\widetilde{U}(\boldsymbol{x})$. Equation 2 implies that $\bar{t}=\widetilde{U}(\boldsymbol{y})-\widetilde{U}(\boldsymbol{x}) \in \mathbb{R} \backslash \mathbb{Z}$, a contradiction to equation 3. Hence, $\mathcal{R}_{\mathbb{Z}} \subsetneq \mathcal{R}_{\mathbb{R}}$. 


\section{The Extension of Ausubel's Auction}

In the extension of Ausubel's auction for discrete heterogeneous goods, there are $N$ bidders and an auctioneer with supply $\boldsymbol{S} \in \mathbb{Z}_{++}^{K}$ of goods to sell. The auction designed is a continuous-time dynamic game. The time is represented by a lexicographically ordered pair $(t, r)$ consisting of a continuously increasing clock $t \in[0, \infty)$, and an increasing integer counter $r \in \mathbb{Z}_{+}$.

The auction starts at clock time $t=0$, an initial price vector $\boldsymbol{p}(0) \in$ $\mathbb{R}_{+}^{K}$, and the counter is initialized to $r=0$. The auctioneer adjusts prices continuously according to the extended tâtonnement algorithm. When the extended ascending algorithm starts at $\boldsymbol{p}(0)$, each bidder reports his demand set at $\boldsymbol{p}(0)$. After this first round of reports, if there are bidders who would like to report new demand sets at the same price vector $\boldsymbol{p}(0)$, the auctioneer increases the counter $r$ by 1 in each round with each new demand set reports from bidders. After the reports are over at prices $\boldsymbol{p}(0)$ (there are finitely many rounds by the no cyclic reports any round assumption stated below), the auctioneer determines the set of goods in excess demand $E_{+}(\boldsymbol{p}(0))$ using the demand reports from the last round at clock time $t=0$. These reports are denoted by $x_{i}(\boldsymbol{p}(0)) \subset X_{i}$ for each bidder $i \in N$. The counter is reset to $r=0$, and the prices of these goods in excess demand are increased at the same rate continuously whereas prices of the rest of the goods remain the same. As prices are being adjusted, bidders may add bundles to their demand set or they may remove bundles from their demand set. Each bidder is required to report his demand set at all price vectors at which he adds a bundle to his demand set.

Steps of the Procedure. Clock time $t \in[0, \infty)$ of the price adjustment is called a step of the procedure if there is a bidder who reports his demand set at $\boldsymbol{p}(t)$.

If a bidder reports his demand set at time $t$, then the auctioneer stops the price adjustment and the clock at time $t$. Then, the counter $r$ is increased, and other bidders are allowed to respond to that bidder's report at the same clock time. This time structure allows bidders to respond to each other's reports at the soonest instant in continuous time (see Ausubel (2004), p.1465, and Simon and Stinchcombe (1989)). I suppress the counter $r$ from the notation of prices $\boldsymbol{p}(t)$ without any confusion because prices will stay the same through out the rounds at the same clock time $t$. The auctioneer increases the counter $r$ by 1 in each round with each new demand set report from bidders. Then, the auctioneer computes the set of goods in excess 
demand $E_{+}(\boldsymbol{p}(t))$ using the demand reports from the last round at clock time $t$, which are denoted by $x_{i}(\boldsymbol{p}(t)) \subset X_{i}$ for each bidder $i \in N$. Next, the auctioneer resumes the price adjustment by increasing prices of all goods in $E_{+}(\boldsymbol{p}(t))$ at the same rate continuously, and holds the prices of the rest of the goods the same. The extended ascending algorithm terminates when the set of goods in excess demand is an empty set. The extended descending algorithm works analogously.

Each bidder observes history $h$ representing the prior play of the game consisting of a finite string of vectors of clock time $t$, price vector $\boldsymbol{p}(t)$ at time $t$, and a profile of demand sets reported at $(t, r)$. In order to simplify the notation, I suppress the round $r$ from the notation without any confusion. The round of the game at clock time $t$ is represented by the order of these vectors. For example, if $h=(0, \boldsymbol{p}(0), \boldsymbol{x}),\left(0, \boldsymbol{p}(0), \boldsymbol{x}^{\prime}\right)$, then $\boldsymbol{x}$ is the round $r=0$ demand reports at clock time $t=0$, and $\boldsymbol{x}^{\prime}$ is the round $r=1$ demand reports at clock time $t=0$. Note that if bidders truthfully report their demand sets, then at each step there will be a single round. The strategy $\sigma_{i}(h) \subset X_{i}$ is a set-valued function of history. The strategy space of $i$ consists of all such functions with the following restriction:

No Cyclic Reports in Any Round. At each $t \in[0, \infty)$, no bidder is allowed to report a demand set twice at any rounds $r$ and $r^{\prime}$ such that $r<r^{\prime}$ if he reports a different demand set at round $r^{\prime \prime}$ such that $r<r^{\prime \prime}<r^{\prime}$.

This restriction does not prevent bidders from bidding sincerely. Since there are finitely many possible demand sets for each bidder, this restriction on strategies also guarantees that the consecutive reporting of bidders at each clock time $t$ lasts finitely many rounds, and the clock resumes its increase at the time where it stopped.

Following Ausubel (2004) and Ausubel (2006), ex post perfect equilibrium is used in the dynamic game defined above:

The strategy profile $\left\{\sigma_{i}\right\}_{i \in N}$ constitutes an ex post perfect equilibrium if for every history $h$, and for every realization of private information $\left\{u_{i}\right\}_{i \in N}$, the profile of continuation strategies $\left\{\sigma_{i}\left(\cdot \mid h, u_{i}\right)\right\}_{i \in N}$ constitutes a Nash equilibrium of the game in which the realization of $\left\{u_{i}\right\}_{i \in N}$ is common knowledge.

The notion of "crediting and debiting," which is introduced in Ausubel's auction and used in the extension proposed here, works as follows: During the price adjustment, if opponents of bidder $j$ lower the quantity they demand of a good in their bids, then it is credited to bidder $i$ at price $\boldsymbol{p}(t)$. On the other hand, if opponents of bidder $j$ rise the quantity they demand of a good, then it is debited from bidder $j$ at price $\boldsymbol{p}(t)$. The auction ends, 
say at time $T \in[0, \infty)$, whenever there is a market clearing allocation in bidders' demand sets at $\boldsymbol{p}(T)$. For each bidder credits and debits are added, monetary transfers are made, and goods are allocated. For an illustration of Ausubel's auction, see the example in Ausubel (2006), pp. 606-607.

For each bidder $i \in N$, his payment is defined as

$$
a_{i}(T)=\boldsymbol{p}(0) \cdot\left[\boldsymbol{S}-\boldsymbol{x}_{-i}(0)\right]-\int_{0}^{T} \boldsymbol{p}(t) \cdot \mathrm{d} \boldsymbol{x}_{-i}(t)
$$

where $\boldsymbol{x}_{\boldsymbol{i}}(t)$ is a bundle bidder $i$ demands at price $\boldsymbol{p}(t)$ and

$$
\boldsymbol{x}_{-\boldsymbol{i}}(t)=\sum_{j \neq i, j \in N} \boldsymbol{x}_{\boldsymbol{j}}(t)
$$

For a detailed discussion of this payment function see Ausubel (2006). In Section 4.3, I show that this payment function is well-defined, and path independent.

\section{The Extended Ascending and Descending Algorithms}

\subsection{Determining the Price Path}

In the extended ascending algorithm and the extended descending algorithm, at each price $\boldsymbol{p}$, given the demand reports of bidders, the set of goods in excess demand is found using the function

$$
L(\boldsymbol{p})=\boldsymbol{p} \cdot \boldsymbol{S}+\sum_{i \in N} V_{i}(\boldsymbol{p})
$$

where $L: \mathbb{R}_{+}^{K} \rightarrow \mathbb{R}$ is a Lyapunov function. Note that this function, by envelope theorem, is minimized at Walrasian equilibrium prices. The following Lemma from Ausubel (2005) shows important properties of the Lyapunov function $L(\cdot)$ of equation 5 .

A function $L: \mathbb{R}_{+}^{K} \rightarrow \mathbb{R}$ is submodular if for each $\boldsymbol{p}, \boldsymbol{p}^{\prime} \in \mathbb{R}_{+}^{K}$

$$
L\left(\boldsymbol{p} \wedge \boldsymbol{p}^{\prime}\right)+L\left(\boldsymbol{p} \vee \boldsymbol{p}^{\prime}\right) \leq L(\boldsymbol{p})+L\left(\boldsymbol{p}^{\prime}\right) .
$$

Lemma 2 (Ausubel (2005)). Under the gross substitutes assumption A4, the Lyapunov function $L(\cdot)$ of equation 5 is a submodular and convex function. 
Proposition 1 below is from Ausubel (2005) and is valid without any change when bidders' values for bundles are real rather than integer. Proposition 1 shows the relationship between the Walrasian equilibrium, the Lyapunov function, and the social surplus.

Proposition 1 (Ausubel (2005)). Suppose that Assumptions A1 - A3 hold, and that a Walrasian equilibrium exists. Then, the set of Walrasian equilibrium price vectors equals the set of minimizers of $L(\cdot)$, and the set of Walrasian equilibria equals the set of all $\left(\boldsymbol{p}^{*}, \boldsymbol{x}^{*}\right)$ such that $\boldsymbol{p}^{*} \in \mathbb{R}_{+}^{K}$ minimizes $L(\cdot)$ and $\left(\boldsymbol{x}_{\boldsymbol{i}}^{*}\right)_{i \in N}$ maximizes

$$
\sum_{i \in N} U_{i}\left(\boldsymbol{x}_{\boldsymbol{i}}\right) \text { subject to } \boldsymbol{x}_{\boldsymbol{i}} \in X_{i} \text { for all } i \in N,
$$

and

$$
\sum_{i \in N} \boldsymbol{x}_{i} \leq \boldsymbol{S}
$$

Corollary. Suppose that Assumptions A1 - A4 hold. Then, the set of Walrasian equilibrium price vectors is a nonempty lattice and there exist the lowest and the highest Walrasian equilibrium price vectors, $\underline{\boldsymbol{p}} \in \mathbb{R}_{+}^{K}$ and $\overline{\boldsymbol{p}} \in \mathbb{R}_{+}^{K}$, respectively.

The Corollary to Proposition 1 is also from Ausubel (2005), but integer properties of the highest and the lowest Walrasian price vectors are dropped as they are not necessarily true when bidders' values for bundles are not restricted to integers. For the proof of the Corollary to Proposition 1, see Ausubel (2005).

The Corollary to Proposition 1 implies that for any economy $\left(\left\{u_{i}(\cdot)\right\}_{i \in N}, \boldsymbol{S}\right)$, there exist Walrasian equilibrium price vectors $\boldsymbol{p} \in \mathbb{R}_{+}^{K}$ and $\overline{\boldsymbol{p}} \in \mathbb{R}_{+}^{K}$ such that if $\boldsymbol{p}^{*} \in \mathbb{R}_{+}^{K}$ is a Walrasian equilibrium price vector, then $\boldsymbol{p} \leq \boldsymbol{p}^{*} \leq \overline{\boldsymbol{p}}$.

The next lemma shows that as a set of prices are increased (decreased) in the extended ascending (descending) algorithm, if a bidder removes a bundle from his demand set, he does so immediately, i.e. as soon as prices start to increase.

Lemma 3. Suppose that Assumptions A1-A2 hold. Let $i \in N, \boldsymbol{x}_{\boldsymbol{i}}, \boldsymbol{x}_{\boldsymbol{i}}^{\prime} \in X_{i}$, and $\boldsymbol{p} \in \mathbb{R}_{+}^{K}$ be such that $U_{i}\left(\boldsymbol{x}_{\boldsymbol{i}}\right)-\boldsymbol{p} \cdot \boldsymbol{x}_{\boldsymbol{i}}=U_{i}\left(\boldsymbol{x}_{\boldsymbol{i}}^{\prime}\right)-\boldsymbol{p} \cdot \boldsymbol{x}_{\boldsymbol{i}}^{\prime}$. 
If there exist $\boldsymbol{\Delta} \in \mathbb{R}_{+}^{K}$ and $\lambda^{\prime}>0$ such that

$$
U_{i}\left(\boldsymbol{x}_{\boldsymbol{i}}\right)-\left(\boldsymbol{p} \mp \lambda^{\prime} \boldsymbol{\Delta}\right) \cdot \boldsymbol{x}_{\boldsymbol{i}}>U_{i}\left(\boldsymbol{x}_{\boldsymbol{i}}^{\prime}\right)-\left(\boldsymbol{p} \mp \lambda^{\prime} \boldsymbol{\Delta}\right) \cdot \boldsymbol{x}_{\boldsymbol{i}}^{\prime},
$$

then for each $\lambda>0$

$$
U_{i}\left(\boldsymbol{x}_{\boldsymbol{i}}\right)-(\boldsymbol{p} \mp \lambda \boldsymbol{\Delta}) \cdot \boldsymbol{x}_{\boldsymbol{i}}>U_{i}\left(\boldsymbol{x}_{\boldsymbol{i}}^{\prime}\right)-(\boldsymbol{p} \mp \lambda \boldsymbol{\Delta}) \cdot \boldsymbol{x}_{\boldsymbol{i}}^{\prime},
$$

and if there exist $\boldsymbol{\Delta} \in \mathbb{R}_{+}^{K}, \boldsymbol{\Delta} \neq \mathbf{0}$ and $\lambda^{\prime}>0$ such that

$$
U_{i}\left(\boldsymbol{x}_{\boldsymbol{i}}\right)-\left(\boldsymbol{p} \mp \lambda^{\prime} \boldsymbol{\Delta}\right) \cdot \boldsymbol{x}_{\boldsymbol{i}}=U_{i}\left(\boldsymbol{x}_{\boldsymbol{i}}^{\prime}\right)-\left(\boldsymbol{p} \mp \lambda^{\prime} \boldsymbol{\Delta}\right) \cdot \boldsymbol{x}_{\boldsymbol{i}}^{\prime},
$$

then for each $\lambda>0$

$$
U_{i}\left(\boldsymbol{x}_{\boldsymbol{i}}\right)-(\boldsymbol{p} \mp \lambda \boldsymbol{\Delta}) \cdot \boldsymbol{x}_{\boldsymbol{i}}=U_{i}\left(\boldsymbol{x}_{\boldsymbol{i}}^{\prime}\right)-(\boldsymbol{p} \mp \lambda \boldsymbol{\Delta}) \cdot \boldsymbol{x}_{\boldsymbol{i}}^{\prime} .
$$

Proof. Suppose that $\boldsymbol{p} \in \mathbb{R}_{+}^{K}$, and $\boldsymbol{x}_{\boldsymbol{i}}, \boldsymbol{x}_{\boldsymbol{i}}^{\prime} \in X_{i}$ such that

$$
U_{i}\left(\boldsymbol{x}_{\boldsymbol{i}}\right)-\boldsymbol{p} \cdot \boldsymbol{x}_{\boldsymbol{i}}=U_{i}\left(\boldsymbol{x}_{\boldsymbol{i}}^{\prime}\right)-\boldsymbol{p} \cdot \boldsymbol{x}_{\boldsymbol{i}}^{\prime} .
$$

Then, by equation 6 , for each $\boldsymbol{\Delta} \geq \mathbf{0}$ and for each $\lambda>0$,

$$
U_{i}\left(\boldsymbol{x}_{\boldsymbol{i}}\right)-(\boldsymbol{p} \mp \lambda \boldsymbol{\Delta}) \cdot \boldsymbol{x}_{\boldsymbol{i}}>U_{i}\left(\boldsymbol{x}_{\boldsymbol{i}}^{\prime}\right)-(\boldsymbol{p} \mp \lambda \boldsymbol{\Delta}) \cdot \boldsymbol{x}_{\boldsymbol{i}}^{\prime}
$$

if and only if

$$
0>\mp \lambda \boldsymbol{\Delta} \cdot\left(\boldsymbol{x}_{\boldsymbol{i}}-\boldsymbol{x}_{\boldsymbol{i}}^{\prime}\right) .
$$

Observe that inequality 7 holds for some $\lambda^{\prime \prime}>0$ if and only if it holds for all $\lambda>0$.

If there exist $\boldsymbol{\Delta} \in \mathbb{R}_{+}^{K}, \boldsymbol{\Delta} \neq \mathbf{0}$ and $\lambda^{\prime}>0$ such that

$$
U_{i}\left(\boldsymbol{x}_{\boldsymbol{i}}\right)-\left(\boldsymbol{p} \mp \lambda^{\prime} \boldsymbol{\Delta}\right) \cdot \boldsymbol{x}_{\boldsymbol{i}}=U_{i}\left(\boldsymbol{x}_{\boldsymbol{i}}^{\prime}\right)-\left(\boldsymbol{p} \mp \lambda^{\prime} \boldsymbol{\Delta}\right) \cdot \boldsymbol{x}_{\boldsymbol{i}}^{\prime},
$$

then by equation 6 ,

$$
\mp \lambda^{\prime} \boldsymbol{\Delta} \cdot\left(\boldsymbol{x}_{\boldsymbol{i}}-\boldsymbol{x}_{\boldsymbol{i}}^{\prime}\right)=0 .
$$

Thus, for each $\lambda>0$,

$$
\mp \lambda \Delta \cdot\left(\boldsymbol{x}_{\boldsymbol{i}}-\boldsymbol{x}_{\boldsymbol{i}}^{\prime}\right)=0 .
$$

Hence, the result follows. 
Suppose that a bidder strictly prefers one bundle to another. If prices are increased (decreased) linearly, then there are two possibilities: either he will keep preferring one to the other, or he will be indifferent between them at a unique price and he will reverse his preferences over these bundles as prices continue to linearly increase (decrease). This is formally stated in the next Lemma.

Lemma 4. Suppose that Assumptions A1-A2 hold. Let $i \in N, \boldsymbol{x}_{\boldsymbol{i}}, \boldsymbol{x}_{\boldsymbol{i}}^{\prime} \in X_{i}$, $\boldsymbol{p} \in \mathbb{R}_{+}^{K}$, and $\boldsymbol{\Delta} \in \mathbb{R}_{+}^{K}$ be such that $U_{i}\left(\boldsymbol{x}_{\boldsymbol{i}}\right)-(\boldsymbol{p} \mp \Delta) \cdot \boldsymbol{x}_{\boldsymbol{i}}>U_{i}\left(\boldsymbol{x}_{\boldsymbol{i}}^{\prime}\right)-(\boldsymbol{p} \mp \Delta) \cdot \boldsymbol{x}_{\boldsymbol{i}}^{\prime}$. Then, one of the following holds:

1. There exists a unique $\lambda_{\boldsymbol{x}_{\boldsymbol{i}}^{\prime}}>0$ such that

$$
U_{i}\left(\boldsymbol{x}_{\boldsymbol{i}}\right)-\left(\boldsymbol{p} \mp \lambda_{\boldsymbol{x}_{\boldsymbol{i}}^{\prime}} \boldsymbol{\Delta}\right) \cdot \boldsymbol{x}_{\boldsymbol{i}}=U_{i}\left(\boldsymbol{x}_{\boldsymbol{i}}^{\prime}\right)-\left(\boldsymbol{p} \mp \lambda_{\boldsymbol{x}_{\boldsymbol{i}}^{\prime}} \Delta\right) \cdot \boldsymbol{x}_{\boldsymbol{i}}^{\prime},
$$

and for each $\lambda \in\left(0, \lambda_{\boldsymbol{x}_{\boldsymbol{i}}^{\prime}}\right)$

$$
U_{i}\left(\boldsymbol{x}_{\boldsymbol{i}}\right)-(\boldsymbol{p} \mp \lambda \boldsymbol{\Delta}) \cdot \boldsymbol{x}_{\boldsymbol{i}}>U_{i}\left(\boldsymbol{x}_{\boldsymbol{i}}^{\prime}\right)-(\boldsymbol{p} \mp \lambda \Delta) \cdot \boldsymbol{x}_{\boldsymbol{i}}^{\prime},
$$

and for each $\lambda>\lambda_{\boldsymbol{x}_{\boldsymbol{i}}^{\prime}}$

$$
U_{i}\left(\boldsymbol{x}_{\boldsymbol{i}}\right)-(\boldsymbol{p} \mp \lambda \boldsymbol{\Delta}) \cdot \boldsymbol{x}_{\boldsymbol{i}}<U_{i}\left(\boldsymbol{x}_{\boldsymbol{i}}^{\prime}\right)-(\boldsymbol{p} \mp \lambda \Delta) \cdot \boldsymbol{x}_{\boldsymbol{i}}^{\prime} .
$$

2. For all $\lambda>0, U_{i}\left(\boldsymbol{x}_{\boldsymbol{i}}\right)-(\boldsymbol{p} \mp \lambda \Delta) \cdot \boldsymbol{x}_{\boldsymbol{i}}>U_{i}\left(\boldsymbol{x}_{\boldsymbol{i}}^{\prime}\right)-(\boldsymbol{p} \mp \lambda \Delta) \cdot \boldsymbol{x}_{\boldsymbol{i}}^{\prime}$.

Proof. If $\mp \boldsymbol{\Delta} \cdot\left(\boldsymbol{x}_{\boldsymbol{i}}-\boldsymbol{x}_{\boldsymbol{i}}^{\prime}\right)>0$ then $U_{i}\left(\boldsymbol{x}_{\boldsymbol{i}}\right)-\left(\boldsymbol{p} \mp \lambda_{\boldsymbol{x}_{\boldsymbol{i}}^{\prime}} \boldsymbol{\Delta}\right) \cdot \boldsymbol{x}_{\boldsymbol{i}}=U_{i}\left(\boldsymbol{x}_{\boldsymbol{i}}^{\prime}\right)-(\boldsymbol{p} \mp$ $\left.\lambda_{\boldsymbol{x}_{\boldsymbol{i}}^{\prime}} \Delta\right) \cdot \boldsymbol{x}_{\boldsymbol{i}}^{\prime}$ for $\lambda_{\boldsymbol{x}_{\boldsymbol{i}}^{\prime}}=\mp \frac{\left(U_{i}\left(\boldsymbol{x}_{\boldsymbol{i}}\right)-\boldsymbol{p} \cdot \boldsymbol{x}_{\boldsymbol{i}}\right)-\left(U_{i}\left(\boldsymbol{x}_{\boldsymbol{i}}^{\prime}\right)-\boldsymbol{p} \cdot \boldsymbol{x}_{\boldsymbol{i}}^{\prime}\right)}{\boldsymbol{\Delta}\left(\boldsymbol{x}_{\boldsymbol{i}}-\boldsymbol{x}_{\boldsymbol{i}}^{\prime}\right)}$. If $\mp \boldsymbol{\Delta} \cdot\left(\boldsymbol{x}_{\boldsymbol{i}}-\boldsymbol{x}_{\boldsymbol{i}}^{\prime}\right) \leq 0$ then $U_{i}\left(\boldsymbol{x}_{\boldsymbol{i}}\right)-(\boldsymbol{p} \mp \lambda \Delta) \cdot \boldsymbol{x}_{\boldsymbol{i}}>U_{i}\left(\boldsymbol{x}_{\boldsymbol{i}}^{\prime}\right)-(\boldsymbol{p} \mp \lambda \Delta) \cdot \boldsymbol{x}_{\boldsymbol{i}}^{\prime}$ for all $\lambda>0$.

Suppose that a bidder $i \in N$ announces his demand set at $Q_{i}(\boldsymbol{p}) \subset X_{i}$ at price vector $\boldsymbol{p} \in \mathbb{R}_{+}^{K}$. I will now show how the auctioneer can determine bundles that will stay in bidder $i$ 's demand set when prices of a subset of goods are all slightly increased (or decreased).

For each $\boldsymbol{p}, \boldsymbol{\Delta} \in \mathbb{R}_{+}^{K}$, a minimal-cost-increase bundle $\widetilde{\boldsymbol{x}}_{\boldsymbol{i}}(\boldsymbol{p}, \boldsymbol{\Delta}) \in Q_{i}(\boldsymbol{p})$ is a bundle bidder $i$ demands at $\boldsymbol{p}$ that has the lowest cost increase when prices increase from $\boldsymbol{p}$ to $\boldsymbol{p}+\boldsymbol{\Delta}$, i.e.

$$
\widetilde{\boldsymbol{x}}_{\boldsymbol{i}}(\boldsymbol{p}, \boldsymbol{\Delta}) \in \underset{x \in Q_{i}(\boldsymbol{p})}{\arg \min }\{\boldsymbol{\Delta} \cdot x\}
$$


Analogously, a maximal-cost-decrease bundle $\widetilde{\boldsymbol{y}}_{\boldsymbol{i}}(\boldsymbol{p}, \boldsymbol{\Delta}) \in Q_{i}(\boldsymbol{p})$ is a bundle bidder $i$ demands at $\boldsymbol{p}$ that has the highest cost decrease when prices decrease from $\boldsymbol{p}$ to $\boldsymbol{p}-\boldsymbol{\Delta}$, i.e.

$$
\widetilde{\boldsymbol{y}}_{\boldsymbol{i}}(\boldsymbol{p}, \boldsymbol{\Delta}) \in \underset{x \in Q_{i}(\boldsymbol{p})}{\arg \max }\{\boldsymbol{\Delta} \cdot x\}
$$

Define

$$
\delta_{i}(\boldsymbol{p})=\frac{1}{2} \inf _{\substack{\boldsymbol{y} \in X_{i} \\ \boldsymbol{y} \notin Q_{i}(\boldsymbol{p})}} \frac{1}{\sum_{k \in K} \bar{x}_{i}^{k}}\left\{\left(U_{i}\left(\widetilde{\boldsymbol{x}}_{\boldsymbol{i}}(\boldsymbol{p}, \boldsymbol{\Delta})\right)-\boldsymbol{p} \cdot \widetilde{\boldsymbol{x}}_{\boldsymbol{i}}(\boldsymbol{p}, \boldsymbol{\Delta})\right)-\left(U_{i}(y)-\boldsymbol{p} \cdot y\right)\right\} .
$$

If $\delta_{i}(\boldsymbol{p})$ is finite, then $\delta_{i}(\boldsymbol{p})$ is the difference between the highest and the second highest utility levels bidder $i$ can achieve from bundles in $X_{i}$ at price $\boldsymbol{p}$ as $X_{i}$ is finite. Hence, for each $\boldsymbol{p}, \boldsymbol{\Delta} \in \mathbb{R}_{+}^{K}$ either

$$
\delta_{i}(\boldsymbol{p})=+\infty
$$

or

$$
\delta_{i}(\boldsymbol{p})>0 .
$$

Note that $\delta_{i}(\boldsymbol{p})$ is infinite if and only if bidder $i$ is indifferent among all bundles at prices $\boldsymbol{p}$.

Construct $\delta(\boldsymbol{p})$ as follows:

$$
\delta(\boldsymbol{p})=\min _{i \in N} \delta_{i}(\boldsymbol{p})
$$

Since there are finitely many bidders in $N$,

$$
\delta(\boldsymbol{p})>0
$$

By the definition of $\delta(\boldsymbol{p})$, for each $\boldsymbol{p} \in \mathbb{R}_{++}$, for each $i \in N$, for each $\boldsymbol{x} \in$ $Q_{i}(\boldsymbol{p})$, and for each $\boldsymbol{y} \in X_{i} \backslash Q_{i}(\boldsymbol{p})$

$$
\left(U_{i}(\boldsymbol{x})-\boldsymbol{p} \cdot \boldsymbol{x}\right)-\left(U_{i}(\boldsymbol{y})-\boldsymbol{p} \cdot \boldsymbol{y}\right)>\delta(\boldsymbol{p}) .
$$

Time $t=0$ is the first step of the extended algorithm. Observe that by the definition of a step, time $t \in(0, \infty)$ is a step of the extended algorithm if there exist $i \in N, \boldsymbol{x}_{\boldsymbol{i}} \in Q_{i}(\boldsymbol{p}(t))$, and $t^{\prime} \in[0, t)$ such that for each $t^{\prime \prime} \in\left(t^{\prime}, t\right)$

$$
\boldsymbol{x}_{\boldsymbol{i}} \notin Q_{i}\left(\boldsymbol{p}\left(t^{\prime \prime}\right)\right) .
$$


Note that steps of the extended algorithm depend on the initial price and preferences of bidders. If all bidders have integer valuations for all bundles, the extended algorithm starts at an integer price vector, and bidders report truthfully, then all steps will be at integer price vectors.

For each $\delta \in \mathbb{R}$, let $\boldsymbol{\delta}^{K}=\left(\delta^{k}\right)_{k \in K}$ denote a $K$-dimensional vector such that $\delta^{k}=\delta$ for all $k \in K$. Proposition 2 shows that the auctioneer can compute each bidder's demand set for all prices in the $K$-dimensional $\delta(\boldsymbol{p})$ wide cube above (below) price vector $\boldsymbol{p}$ using that bidder's demand set at $p$.

Proposition 2. Suppose that Assumptions $A 1-A 2$ hold. For each $i \in N$, for each $\boldsymbol{p} \in \mathbb{R}_{+}^{K}$, for each $\boldsymbol{\Delta} \in \mathbb{R}_{+}^{K}$, and for each $\lambda \in \mathbb{R}_{++}$such that $\mathbf{0} \lesseqgtr \boldsymbol{\Delta} \leq \boldsymbol{\delta}(\boldsymbol{p})^{K}$, and $\mathbf{0} \lesseqgtr \lambda \boldsymbol{\Delta} \leq \boldsymbol{\delta}(\boldsymbol{p})^{K}$

$$
Q_{i}(\boldsymbol{p}+\lambda \boldsymbol{\Delta})=\left\{\widetilde{\boldsymbol{x}}_{\boldsymbol{i}}(\boldsymbol{p}, \boldsymbol{\Delta}) \in Q_{i}(\boldsymbol{p})\right\},
$$

and

$$
Q_{i}(\boldsymbol{p}-\lambda \boldsymbol{\Delta})=\left\{\widetilde{\boldsymbol{y}}_{\boldsymbol{i}}(\boldsymbol{p}, \boldsymbol{\Delta}) \in Q_{i}(\boldsymbol{p})\right\} .
$$

Proof. Let $i \in N, \boldsymbol{p} \in \mathbb{R}_{+}^{K}$, and $\boldsymbol{\Delta} \in \mathbb{R}_{+}^{K}$ such that $\mathbf{0} \lesseqgtr \boldsymbol{\Delta} \leq \boldsymbol{\delta}(\boldsymbol{p})^{K}$. Let $\boldsymbol{y} \in X_{i}$.

Claim. If $\boldsymbol{y} \notin\left\{\widetilde{\boldsymbol{x}}_{\boldsymbol{i}}(\boldsymbol{p}, \boldsymbol{\Delta}) \in Q_{i}(\boldsymbol{p})\right\}$, then for each $\lambda \in \mathbb{R}_{++}$such that $\mathbf{0} \lesseqgtr$ $\lambda \boldsymbol{\Delta} \leq \boldsymbol{\delta}(\boldsymbol{p})^{K}$

$$
U_{i}\left(\widetilde{\boldsymbol{x}}_{\boldsymbol{i}}(\boldsymbol{p}, \boldsymbol{\Delta})\right)-(\boldsymbol{p}+\lambda \boldsymbol{\Delta}) \cdot \widetilde{\boldsymbol{x}}_{\boldsymbol{i}}(\boldsymbol{p}, \boldsymbol{\Delta})>U_{i}(\boldsymbol{y})-(\boldsymbol{p}+\lambda \boldsymbol{\Delta}) \cdot \boldsymbol{y} .
$$

Proof of the Claim. There are two cases to consider:

Case 1. If $\boldsymbol{y} \in Q_{i}(\boldsymbol{p}) \backslash\left\{\widetilde{\boldsymbol{x}}_{\boldsymbol{i}}(\boldsymbol{p}, \boldsymbol{\Delta}) \in Q_{i}(\boldsymbol{p})\right\}$, then for each $\lambda \in \mathbb{R}_{++}$such that $\mathbf{0} \lesseqgtr \lambda \boldsymbol{\Delta} \leq \boldsymbol{\delta}(\boldsymbol{p})^{K}$

$$
U_{i}\left(\widetilde{\boldsymbol{x}}_{\boldsymbol{i}}(\boldsymbol{p}, \boldsymbol{\Delta})\right)-(\boldsymbol{p}+\lambda \boldsymbol{\Delta}) \cdot \widetilde{\boldsymbol{x}}_{\boldsymbol{i}}(\boldsymbol{p}, \boldsymbol{\Delta})>U_{i}(\boldsymbol{y})-(\boldsymbol{p}+\lambda \boldsymbol{\Delta}) \cdot \boldsymbol{y} .
$$

Case 2. If $\boldsymbol{y} \notin Q_{i}(\boldsymbol{p})$, then

$$
U_{i}\left(\widetilde{\boldsymbol{x}}_{\boldsymbol{i}}(\boldsymbol{p}, \boldsymbol{\Delta})\right)-\boldsymbol{p} \cdot \widetilde{\boldsymbol{x}}_{\boldsymbol{i}}(\boldsymbol{p}, \boldsymbol{\Delta})>U_{i}(\boldsymbol{y})-\boldsymbol{p} \cdot \boldsymbol{y}
$$

Let $\lambda \in \mathbb{R}_{++}$such that $\mathbf{0} \lesseqgtr \lambda \boldsymbol{\Delta} \leq \boldsymbol{\delta}(\boldsymbol{p})^{K}$. Observe that as $\boldsymbol{\Delta} \geq \mathbf{0}$, and $\widetilde{\boldsymbol{x}}_{\boldsymbol{i}}(\boldsymbol{p}, \boldsymbol{\Delta}), \boldsymbol{y} \in X_{i}$

$$
\lambda \boldsymbol{\Delta} \cdot\left(\widetilde{\boldsymbol{x}}_{\boldsymbol{i}}(\boldsymbol{p}, \boldsymbol{\Delta})-\boldsymbol{y}\right) \leq \lambda \boldsymbol{\Delta} \cdot\left(\bar{x}_{i}^{k}\right)_{k \in K}=\lambda \sum_{k \in K}\left(\Delta^{k} \bar{x}_{i}^{k}\right)
$$


Hence,

$$
\lambda \boldsymbol{\Delta} \cdot\left(\widetilde{\boldsymbol{x}}_{\boldsymbol{i}}(\boldsymbol{p}, \boldsymbol{\Delta})-\boldsymbol{y}\right) \leq \lambda \sum_{k \in K}\left(\Delta^{k} \bar{x}_{i}^{k}\right)
$$

If there does not exist $\lambda_{y}>0$ such that

$$
U_{i}\left(\widetilde{\boldsymbol{x}}_{\boldsymbol{i}}(\boldsymbol{p}, \boldsymbol{\Delta})\right)-\left(\boldsymbol{p}+\lambda_{y} \boldsymbol{\Delta}\right) \cdot \widetilde{\boldsymbol{x}}_{\boldsymbol{i}}(\boldsymbol{p}, \boldsymbol{\Delta})=U_{i}(\boldsymbol{y})-\left(\boldsymbol{p}+\lambda_{y} \boldsymbol{\Delta}\right) \cdot \boldsymbol{y},
$$

then by Lemma 4

$$
U_{i}\left(\widetilde{\boldsymbol{x}}_{\boldsymbol{i}}(\boldsymbol{p}, \boldsymbol{\Delta})\right)-(\boldsymbol{p}+\lambda \boldsymbol{\Delta}) \cdot \widetilde{\boldsymbol{x}}_{\boldsymbol{i}}(\boldsymbol{p}, \boldsymbol{\Delta})>U_{i}(\boldsymbol{y})-(\boldsymbol{p}+\lambda \boldsymbol{\Delta}) \cdot \boldsymbol{y} .
$$

If there exists such $\lambda_{y}>0$, then, as $\left(U_{i}\left(\widetilde{\boldsymbol{x}}_{\boldsymbol{i}}(\boldsymbol{p}, \boldsymbol{\Delta})\right)-\boldsymbol{p} \cdot \widetilde{\boldsymbol{x}}_{\boldsymbol{i}}(\boldsymbol{p}, \boldsymbol{\Delta})\right)-\left(U_{i}(\boldsymbol{y})-\right.$ $\boldsymbol{p} \cdot \boldsymbol{y})=\left(\widetilde{\boldsymbol{x}}_{\boldsymbol{i}}(\boldsymbol{p}, \boldsymbol{\Delta})-\boldsymbol{y}\right) \cdot \lambda_{y} \boldsymbol{\Delta}$, and by the definition of $\delta(\boldsymbol{p})$, for each $k \in K$

$$
\lambda \Delta^{k} \leq \delta(\boldsymbol{p})<\lambda_{y} \boldsymbol{\Delta} \cdot\left(\widetilde{\boldsymbol{x}}_{\boldsymbol{i}}(\boldsymbol{p}, \boldsymbol{\Delta})-y\right) \frac{1}{\sum_{k \in K} \bar{x}_{i}^{k}} .
$$

Thus, by multiplying with $\bar{x}_{i}^{k}$

$$
\lambda \Delta^{k} \bar{x}_{i}^{k}<\lambda_{y} \boldsymbol{\Delta} \cdot\left(\widetilde{\boldsymbol{x}}_{\boldsymbol{i}}(\boldsymbol{p}, \boldsymbol{\Delta})-y\right) \frac{\bar{x}_{i}^{k}}{\sum_{k \in K} \bar{x}_{i}^{k}},
$$

and summing over $k \in K$

$$
\lambda \sum_{k \in K} \Delta^{k} \bar{x}_{i}^{k}<\lambda_{y} \boldsymbol{\Delta} \cdot\left(\widetilde{\boldsymbol{x}}_{\boldsymbol{i}}(\boldsymbol{p}, \boldsymbol{\Delta})-y\right) .
$$

Inequalities 12 and 14 imply that

$$
\lambda \boldsymbol{\Delta} \cdot\left(\widetilde{\boldsymbol{x}}_{\boldsymbol{i}}(\boldsymbol{p}, \boldsymbol{\Delta})-y\right)<\lambda_{y} \boldsymbol{\Delta} \cdot\left(\widetilde{\boldsymbol{x}}_{\boldsymbol{i}}(\boldsymbol{p}, \boldsymbol{\Delta})-y\right) .
$$

So, by the definition of $\lambda_{y}$

$$
\lambda \boldsymbol{\Delta} \cdot\left(\widetilde{\boldsymbol{x}}_{\boldsymbol{i}}(\boldsymbol{p}, \boldsymbol{\Delta})-y\right)<\left(U_{i}\left(\widetilde{\boldsymbol{x}}_{\boldsymbol{i}}(\boldsymbol{p}, \boldsymbol{\Delta})\right)-\boldsymbol{p} \cdot \widetilde{\boldsymbol{x}}_{\boldsymbol{i}}(\boldsymbol{p}, \boldsymbol{\Delta})\right)-\left(U_{i}(\boldsymbol{y})-\boldsymbol{p} \cdot \boldsymbol{y}\right) .
$$

Hence,

$$
U_{i}\left(\widetilde{\boldsymbol{x}}_{\boldsymbol{i}}(\boldsymbol{p}, \boldsymbol{\Delta})\right)-(\boldsymbol{p}+\lambda \boldsymbol{\Delta}) \cdot \widetilde{\boldsymbol{x}}_{\boldsymbol{i}}(\boldsymbol{p}, \boldsymbol{\Delta})>U_{i}(\boldsymbol{y})-(\boldsymbol{p}+\lambda \boldsymbol{\Delta}) \cdot \boldsymbol{y} .
$$

This completes the proof of the Claim. 
By the definition of $\widetilde{\boldsymbol{x}}_{\boldsymbol{i}}(\boldsymbol{p}, \boldsymbol{\Delta})$, for all minimal-cost-increase bundles $\widetilde{\boldsymbol{x}}_{\boldsymbol{i}}(\boldsymbol{p}, \boldsymbol{\Delta})$ and $\widetilde{\boldsymbol{x}}_{\boldsymbol{i}}^{\prime}(\boldsymbol{p}, \boldsymbol{\Delta})$, and for each $\lambda>0$,

$$
U_{i}\left(\widetilde{\boldsymbol{x}}_{\boldsymbol{i}}^{\prime}(\boldsymbol{p}, \boldsymbol{\Delta})\right)-(\boldsymbol{p}+\lambda \boldsymbol{\Delta}) \cdot \widetilde{\boldsymbol{x}}_{\boldsymbol{i}}^{\prime}(\boldsymbol{p}, \boldsymbol{\Delta})=U_{i}\left(\widetilde{\boldsymbol{x}}_{\boldsymbol{i}}(\boldsymbol{p}, \boldsymbol{\Delta})\right)-(\boldsymbol{p}+\lambda \boldsymbol{\Delta}) \cdot \widetilde{\boldsymbol{x}}_{\boldsymbol{i}}(\boldsymbol{p}, \boldsymbol{\Delta}) .
$$

Equations 11, 13, 17 and 16 imply that

$$
Q_{i}(\boldsymbol{p}+\lambda \boldsymbol{\Delta})=\left\{\widetilde{\boldsymbol{x}}_{\boldsymbol{i}}(\boldsymbol{p}, \boldsymbol{\Delta}) \in Q_{i}(\boldsymbol{p})\right\} .
$$

Analogously it can be shown that

$$
Q_{i}(\boldsymbol{p}-\lambda \boldsymbol{\Delta})=\left\{\widetilde{\boldsymbol{y}}_{\boldsymbol{i}}(\boldsymbol{p}, \boldsymbol{\Delta}) \in Q_{i}(\boldsymbol{p})\right\} .
$$

For each $\delta \in \mathbb{R}$, and for each subset of goods $E \subset K$, let $\boldsymbol{\delta}^{E}=\left(\delta^{k}\right)_{k \in K}$ denote a $K$-dimensional vector such that $\delta^{k}=0$ if $k \notin E$, and $\delta^{k}=\delta$ if $k \in E$.

As prices change, bidders' demand sets change: a bidder may remove a bundle from his demand set, or add a bundle to his demand set. Lemma 5 shows a relationship between bundles in the demand set and those added during the price adjustment.

Lemma 5. Suppose that Assumptions $A 1-$ A4 hold. Let $i \in N, \boldsymbol{p}, \boldsymbol{p}^{\prime} \in \mathbb{R}_{+}^{K}$ such that $\boldsymbol{p} \leq \boldsymbol{p}^{\prime}$,

$$
Q_{i}\left(\boldsymbol{p}^{\prime}\right) \backslash Q_{i}(\boldsymbol{p}) \neq \emptyset
$$

and

$$
Q_{i}\left(\boldsymbol{p}^{\prime}\right) \cap Q_{i}(\boldsymbol{p}) \neq \emptyset .
$$

Then, there exist

$$
\boldsymbol{x}_{\boldsymbol{i}}^{\prime} \in Q_{i}\left(\boldsymbol{p}^{\prime}\right) \backslash Q_{i}(\boldsymbol{p}),
$$

and

$$
\boldsymbol{x}_{\boldsymbol{i}} \in Q_{i}\left(\boldsymbol{p}^{\prime}\right) \cap Q_{i}(\boldsymbol{p})
$$

such that

$$
\#\left(\boldsymbol{x}_{\boldsymbol{i}} \backslash \boldsymbol{x}_{\boldsymbol{i}}^{\prime}\right)=1 \text { and } \#\left(\boldsymbol{x}_{\boldsymbol{i}}^{\prime} \backslash \boldsymbol{x}_{\boldsymbol{i}}\right) \leq 1 \text {. }
$$

Moreover, there exists a unique $k \in K$ such that

$$
x_{i}^{\prime k}=x_{i}^{k}-1 .
$$


Also,

$$
p_{k}<p_{k}^{\prime} \text {. }
$$

On the other hand, if there exists $k^{\prime} \in K$ such that $x_{i}^{\prime k^{\prime}}=x_{i}^{k^{\prime}}+1$, then

$$
p_{k^{\prime}}^{\prime}-p_{k^{\prime}}<p_{k}^{\prime}-p_{k}
$$

If there exists $E \subset K$ and $\delta \in \mathbb{R}_{++}$such that $\boldsymbol{p}^{\prime}=\boldsymbol{p}+\boldsymbol{\delta}^{E}$, then

$$
k \in E,
$$

and

$$
k^{\prime} \notin E \text { if there exists } k^{\prime} \in K \text { such that } x_{i}^{\prime k^{\prime}}=x_{i}^{k^{\prime}}+1 \text {. }
$$

Proof. Define $\boldsymbol{\Delta}=\boldsymbol{p}^{\prime}-\boldsymbol{p}$. By Lemma 4, equation 18 implies that there does not exist $\lambda \in(0,1)$ such that

$$
Q_{i}(\boldsymbol{p}+\lambda \boldsymbol{\Delta}) \backslash Q_{i}(\boldsymbol{p}) \neq \emptyset,
$$

i.e. as prices are increased linearly from $\boldsymbol{p}$ to $\boldsymbol{p}^{\prime}, \boldsymbol{p}^{\prime}$ is the first price vector reached at which a new bundle is added to the demand set.

Construction of $\boldsymbol{x}_{\boldsymbol{i}}^{\prime}$. Let $\boldsymbol{x}_{\boldsymbol{i}}^{\prime} \in Q_{i}\left(\boldsymbol{p}^{\prime}\right) \backslash Q_{i}(\boldsymbol{p})$ such that for each $\boldsymbol{y} \in$ $Q_{i}\left(\boldsymbol{p}^{\prime}\right) \backslash Q_{i}(\boldsymbol{p})$

$$
U_{i}\left(\boldsymbol{x}_{\boldsymbol{i}}^{\prime}\right)-\boldsymbol{p} \cdot \boldsymbol{x}_{\boldsymbol{i}}^{\prime} \geq U_{i}(\boldsymbol{y})-\boldsymbol{p} \cdot \boldsymbol{y} .
$$

$\boldsymbol{x}_{\boldsymbol{i}}^{\prime}$ is a bundle bidder $i$ adds to his demand set at $\boldsymbol{p}^{\prime}$ such that bidder $i$ prefers this bundle at $\boldsymbol{p}$ to all other bundles added at $\boldsymbol{p}^{\prime}$. Such $\boldsymbol{x}_{\boldsymbol{i}}^{\prime}$ exists because $X_{i}$ is finite.

Construction of $\boldsymbol{x}_{\boldsymbol{i}}$. By Lemma 4, for each $\boldsymbol{y} \in Q_{i}\left(\boldsymbol{p}^{\prime}\right) \backslash Q_{i}(\boldsymbol{p})$, and for each $\lambda \in[0,1]$

$$
U_{i}\left(\boldsymbol{x}_{\boldsymbol{i}}^{\prime}\right)-(\boldsymbol{p}+\lambda \boldsymbol{\Delta}) \cdot \boldsymbol{x}_{\boldsymbol{i}}^{\prime} \geq U_{i}(\boldsymbol{y})-(\boldsymbol{p}+\lambda \boldsymbol{\Delta}) \cdot \boldsymbol{y} .
$$

As $X_{i}$ is finite, by Lemma 4 , there exists $\lambda^{\prime} \in(0,1)$ such that for each $\lambda \in\left(\lambda^{\prime}, 1\right)$, and $\boldsymbol{y} \in X_{i}$, if

$$
U_{i}(\boldsymbol{y})-(\boldsymbol{p}+\lambda \boldsymbol{\Delta}) \cdot \boldsymbol{y}>U_{i}\left(\boldsymbol{x}_{\boldsymbol{i}}^{\prime}\right)-(\boldsymbol{p}+\lambda \boldsymbol{\Delta}) \cdot \boldsymbol{x}_{\boldsymbol{i}}^{\prime}
$$

then

$$
\boldsymbol{y} \in Q_{i}(\boldsymbol{p}+\lambda \boldsymbol{\Delta}) .
$$

In words, there exists a price $\boldsymbol{p}+\lambda^{\prime} \boldsymbol{\Delta}$ such that at all prices reached between $\boldsymbol{p}+\lambda^{\prime} \boldsymbol{\Delta}$ and $\boldsymbol{p}^{\prime}$, there is no bundle both strictly preferred to $\boldsymbol{x}_{\boldsymbol{i}}^{\prime}$ and not in 
bidder $i$ 's demand set. Hence, by the single-improvement property (implied by Assumptions the gross substitutes $A 4$ and monotonicity $A 3$, see Lemma 2 in Gul and Stacchetti (1999)), there exists

$$
\boldsymbol{x}_{\boldsymbol{i}} \in Q_{i}(\boldsymbol{p}+\lambda \boldsymbol{\Delta})
$$

such that

$$
\#\left(\boldsymbol{x}_{\boldsymbol{i}} \backslash \boldsymbol{x}_{\boldsymbol{i}}^{\prime}\right) \leq 1 \text { and } \#\left(\boldsymbol{x}_{\boldsymbol{i}}^{\prime} \backslash \boldsymbol{x}_{\boldsymbol{i}}\right) \leq 1 \text {. }
$$

Since the first new bundle is added at $\boldsymbol{p}^{\prime}$ to bidder $i$ 's demand set as prices are increased linearly from $\boldsymbol{p}$ to $\boldsymbol{p}^{\prime}$, by Proposition 2,

$$
Q_{i}(\boldsymbol{p}+\lambda \boldsymbol{\Delta})=Q_{i}(\boldsymbol{p}) \cap Q_{i}\left(\boldsymbol{p}^{\prime}\right) .
$$

Therefore,

$$
\boldsymbol{x}_{\boldsymbol{i}} \in Q_{i}(\boldsymbol{p}) \cap Q_{i}\left(\boldsymbol{p}^{\prime}\right),
$$

and

$$
\#\left(\boldsymbol{x}_{\boldsymbol{i}} \backslash \boldsymbol{x}_{\boldsymbol{i}}^{\prime}\right) \leq 1 \text { and } \#\left(\boldsymbol{x}_{\boldsymbol{i}}^{\prime} \backslash \boldsymbol{x}_{\boldsymbol{i}}\right) \leq 1 .
$$

So, $\boldsymbol{x}_{\boldsymbol{i}}$ is a bundle in bidder $i$ 's demand sets at all prices reached between $\boldsymbol{p}$ and $\boldsymbol{p}^{\prime}$ such that $\boldsymbol{x}_{\boldsymbol{i}}^{\prime}$ can be constructed by adding at most a unit of a good and removing at most a unit of another good to $\boldsymbol{x}_{\boldsymbol{i}}$.

$$
\begin{aligned}
\text { As } U_{i}\left(\boldsymbol{x}_{\boldsymbol{i}}^{\prime}\right)-(\boldsymbol{p}+\boldsymbol{\Delta}) \cdot \boldsymbol{x}_{\boldsymbol{i}}^{\prime}=U_{i}\left(\boldsymbol{x}_{\boldsymbol{i}}\right)-(\boldsymbol{p}+\boldsymbol{\Delta}) \cdot \boldsymbol{x}_{\boldsymbol{i}}, \\
\quad-\left(U_{i}\left(\boldsymbol{x}_{\boldsymbol{i}}^{\prime}\right)-\boldsymbol{p} \cdot \boldsymbol{x}_{\boldsymbol{i}}^{\prime}\right)+\left(U_{i}\left(\boldsymbol{x}_{\boldsymbol{i}}\right)-\boldsymbol{p} \cdot \boldsymbol{x}_{\boldsymbol{i}}\right)=\left(\boldsymbol{x}_{\boldsymbol{i}}-\boldsymbol{x}_{\boldsymbol{i}}^{\prime}\right) \cdot \boldsymbol{\Delta} .
\end{aligned}
$$

Since $\boldsymbol{x}_{\boldsymbol{i}} \in Q_{i}(\boldsymbol{p})$ and $\boldsymbol{x}_{\boldsymbol{i}}^{\prime} \notin Q_{i}(\boldsymbol{p})$,

$$
\left(\boldsymbol{x}_{\boldsymbol{i}}-\boldsymbol{x}_{\boldsymbol{i}}^{\prime}\right) \cdot \Delta>0 .
$$

Therefore, by equations 19, there exists a unique $k \in K$ such that $x_{i}^{\prime k}=x_{i}^{k}-1$. Observe that $\Delta_{k}=p_{k}^{\prime}-p_{k}>0$. Moreover, if there exists $k^{\prime} \in K$ such that $x_{i}^{\prime k^{\prime}}=x_{i}^{k^{\prime}}+1$, then $\Delta_{k}-\Delta_{k^{\prime}}>0$ implying $p_{k^{\prime}}^{\prime}-p_{k^{\prime}}<p_{k}^{\prime}-p_{k}$. Otherwise, $\Delta_{k}>0$ implying $p_{k}<p_{k}^{\prime}$. The rest of the proof follows trivially.

Lemma 6 explains the relationship between each bidder's demand set on the corners of $\delta(\boldsymbol{p})$-wide cube above (below) price vector $\boldsymbol{p}$ and his demand sets on the edges of that cube. 
Lemma 6. Suppose that Assumptions $A 1-A 4$ hold. For each $\boldsymbol{p} \in \mathbb{R}_{+}^{K}$, for each $\delta \in[0, \delta(\boldsymbol{p})]$, for each $E \subset K$, for each $i \in N$, and for each $\boldsymbol{\Delta} \geq \mathbf{0}$ such that for each $k \in E \Delta^{k}=0$, and for each $\lambda \in \mathbb{R}_{+}$such that $\mathbf{0} \leq \lambda \boldsymbol{\Delta} \leq \boldsymbol{\delta}^{K}$

$$
\left\{\widetilde{\boldsymbol{x}}_{\boldsymbol{i}}\left(\boldsymbol{p}+\boldsymbol{\delta}^{E}, \boldsymbol{\Delta}\right) \in Q_{i}\left(\boldsymbol{p}+\boldsymbol{\delta}^{E}\right)\right\}=Q_{i}\left(\boldsymbol{p}+\boldsymbol{\delta}^{E}+\lambda \boldsymbol{\Delta}\right),
$$

and

$$
\left\{\widetilde{\boldsymbol{y}}_{\boldsymbol{i}}\left(\boldsymbol{p}-\boldsymbol{\delta}^{E}, \boldsymbol{\Delta}\right) \in Q_{i}\left(\boldsymbol{p}-\boldsymbol{\delta}^{E}\right)\right\}=Q_{i}\left(\boldsymbol{p}-\boldsymbol{\delta}^{E}-\lambda \boldsymbol{\Delta}\right) .
$$

Proof. Consider the equation 20. Suppose on the contrary that there exist $\boldsymbol{p} \in \mathbb{R}_{+}^{K}, \delta \in[0, \delta(\boldsymbol{p})], E \subset K, i \in N, \lambda \in \mathbb{R}_{+}, \boldsymbol{\Delta} \in \mathbb{R}_{+}^{K}$ and $\widetilde{\boldsymbol{x}}_{\boldsymbol{i}}\left(\boldsymbol{p}+\boldsymbol{\delta}^{E}, \boldsymbol{\Delta}\right)$ such that $\mathbf{0} \leq \lambda \boldsymbol{\Delta} \leq \boldsymbol{\delta}^{K}$, and for each $k \in E \Delta^{k}=0$, and equation 20 does not hold. Then, either there exists $\widetilde{\boldsymbol{x}}_{\boldsymbol{i}}\left(\boldsymbol{p}+\boldsymbol{\delta}^{E}, \boldsymbol{\Delta}\right) \in Q_{i}\left(\boldsymbol{p}+\boldsymbol{\delta}^{E}\right)$ such that

$$
\widetilde{\boldsymbol{x}}_{\boldsymbol{i}}\left(\boldsymbol{p}+\boldsymbol{\delta}^{E}, \boldsymbol{\Delta}\right) \notin Q_{i}\left(\boldsymbol{p}+\boldsymbol{\delta}^{E}+\lambda \boldsymbol{\Delta}\right),
$$

or there exists

$$
\boldsymbol{x}_{\boldsymbol{i}} \in Q_{i}\left(\boldsymbol{p}+\boldsymbol{\delta}^{E}+\lambda \boldsymbol{\Delta}\right) \backslash\left\{\widetilde{\boldsymbol{x}}_{\boldsymbol{i}}\left(\boldsymbol{p}+\boldsymbol{\delta}^{E}, \boldsymbol{\Delta}\right) \in Q_{i}\left(\boldsymbol{p}+\boldsymbol{\delta}^{E}\right)\right\} .
$$

Suppose that equation 21 holds. If $\delta=0$ or $\lambda \boldsymbol{\Delta}=\mathbf{0}$, then equation 21 contradicts to the definition of $\widetilde{\boldsymbol{x}}_{\boldsymbol{i}}(\cdot, \cdot)$. So, suppose that $\delta>0$ and $\lambda \boldsymbol{\Delta} \gtrless \mathbf{0}$. By Lemma 3, equation 21 implies that

$$
\left\{\widetilde{\boldsymbol{x}}_{\boldsymbol{i}}\left(\boldsymbol{p}+\boldsymbol{\delta}^{E}, \boldsymbol{\Delta}\right) \in Q_{i}\left(\boldsymbol{p}+\boldsymbol{\delta}^{E}\right)\right\} \cap Q_{i}\left(\boldsymbol{p}+\boldsymbol{\delta}^{E}+\lambda \boldsymbol{\Delta}\right)=\emptyset,
$$

which means that all minimal cost bundles are dropped from the demand set at the same price vector which is between $\boldsymbol{p}+\boldsymbol{\delta}^{E}$ and $\boldsymbol{p}+\boldsymbol{\delta}^{E}+\lambda \boldsymbol{\Delta}$. Hence, by Lemma 3 again,

$$
Q_{i}\left(\boldsymbol{p}+\boldsymbol{\delta}^{E}\right) \cap Q_{i}\left(\boldsymbol{p}+\boldsymbol{\delta}^{E}+\lambda \boldsymbol{\Delta}\right)=\emptyset
$$

which implies equation 22. So, it is sufficient to show that equation 22 results in a contradiction.

Define

$$
\begin{aligned}
\underline{\lambda}=\min & \left\{\lambda^{\prime} \in \mathbb{R}_{++}: \text {there exists } \boldsymbol{x}_{\boldsymbol{i}}^{\prime} \in X_{i} \backslash Q_{i}\left(\boldsymbol{p}+\boldsymbol{\delta}^{E}\right)\right. \text { such that } \\
& U_{i}\left(\boldsymbol{x}_{\boldsymbol{i}}^{\prime}\right)-\left(\boldsymbol{p}+\boldsymbol{\delta}^{E}+\lambda^{\prime} \boldsymbol{\Delta}\right) \cdot \boldsymbol{x}_{\boldsymbol{i}}^{\prime} \\
& \left.=U_{i}\left(\widetilde{\boldsymbol{x}}_{\boldsymbol{i}}\left(\boldsymbol{p}+\boldsymbol{\delta}^{E}, \boldsymbol{\Delta}\right)\right)-\left(\boldsymbol{p}+\boldsymbol{\delta}^{E}+\lambda^{\prime} \boldsymbol{\Delta}\right) \cdot \widetilde{\boldsymbol{x}}_{\boldsymbol{i}}\left(\boldsymbol{p}+\boldsymbol{\delta}^{E}, \boldsymbol{\Delta}\right)\right\} .
\end{aligned}
$$


Note that $\boldsymbol{p}+\boldsymbol{\delta}^{E}+\underline{\lambda} \boldsymbol{\Delta}$ is the first price vector reached at which bidder $i$ adds a bundle to his demand set as prices increase linearly from $\boldsymbol{p}+\boldsymbol{\delta}^{E}$ to $\boldsymbol{p}+\boldsymbol{\delta}^{E}+\lambda \boldsymbol{\Delta}$. Let $\boldsymbol{x}_{\boldsymbol{i}} \in X_{i}$ be a bundle which bidder $i$ adds to his demand set at $\boldsymbol{p}+\boldsymbol{\delta}^{E}+\underline{\lambda} \boldsymbol{\Delta}$ such that $\underline{\boldsymbol{x}}_{\boldsymbol{i}}$ is preferred at all prices between $\boldsymbol{p}+\boldsymbol{\delta}^{E}$ and $\boldsymbol{p}+\boldsymbol{\delta}^{E}+\lambda \boldsymbol{\Delta}$ to all other bundles which bidder $i$ adds to his demand set at $\boldsymbol{p}+\boldsymbol{\delta}^{E}+\underline{\lambda} \boldsymbol{\Delta}$. Since $X_{i}$ is finite, by Lemma 4 and equation $22, \underline{\lambda}$ is well-defined, and $\underline{\lambda}>0$. Observe that

$$
\underline{\lambda}<\lambda,
$$

and hence $\mathbf{0} \lesseqgtr \underline{\lambda} \boldsymbol{\Delta} \ll \boldsymbol{\delta}^{K}$. So, $\underline{\boldsymbol{x}}_{\boldsymbol{i}} \in X_{i}$ is such that

$$
\begin{aligned}
U_{i}\left(\underline{\boldsymbol{x}}_{i}\right)-\left(\boldsymbol{p}+\boldsymbol{\delta}^{E}+\underline{\lambda} \boldsymbol{\Delta}\right) \cdot \underline{\boldsymbol{x}}_{i}= & U_{i}\left(\widetilde{\boldsymbol{x}}_{\boldsymbol{i}}\left(\boldsymbol{p}+\boldsymbol{\delta}^{E}, \boldsymbol{\Delta}\right)\right) \\
& -\left(\boldsymbol{p}+\boldsymbol{\delta}^{E}+\underline{\lambda} \boldsymbol{\Delta}\right) \cdot \widetilde{\boldsymbol{x}}_{\boldsymbol{i}}\left(\boldsymbol{p}+\boldsymbol{\delta}^{E}, \boldsymbol{\Delta}\right),
\end{aligned}
$$

and for each $\lambda^{\prime} \in[0, \underline{\lambda}]$, and for each $\boldsymbol{x}_{\boldsymbol{i}} \in Q_{i}\left(\boldsymbol{p}+\boldsymbol{\delta}^{E}+\underline{\lambda} \boldsymbol{\Delta}\right) \backslash Q_{i}\left(\boldsymbol{p}+\boldsymbol{\delta}^{E}\right)$

$$
U_{i}\left(\underline{\boldsymbol{x}}_{i}\right)-\left(\boldsymbol{p}+\boldsymbol{\delta}^{E}+\lambda^{\prime} \boldsymbol{\Delta}\right) \cdot \underline{\boldsymbol{x}}_{i} \geq U_{i}\left(\boldsymbol{x}_{\boldsymbol{i}}\right)-\left(\boldsymbol{p}+\boldsymbol{\delta}^{E}+\lambda^{\prime} \boldsymbol{\Delta}\right) \cdot \boldsymbol{x}_{\boldsymbol{i}} .
$$

Note that $\underline{\boldsymbol{x}}_{\boldsymbol{i}} \notin Q_{i}\left(\boldsymbol{p}+\boldsymbol{\delta}^{E}\right)$, and by Lemma 5 , there exists

$$
\widetilde{\boldsymbol{x}}_{\boldsymbol{i}}\left(\boldsymbol{p}+\boldsymbol{\delta}^{E}, \boldsymbol{\Delta}\right) \in Q_{i}\left(\boldsymbol{p}+\boldsymbol{\delta}^{E}+\underline{\lambda} \boldsymbol{\Delta}\right)
$$

such that

$$
\#\left(\widetilde{\boldsymbol{x}}_{\boldsymbol{i}}\left(\boldsymbol{p}+\boldsymbol{\delta}^{E}, \boldsymbol{\Delta}\right) \backslash \underline{\boldsymbol{x}}_{\boldsymbol{i}}\right)=1
$$

and

$$
\#\left(\underline{\boldsymbol{x}}_{\boldsymbol{i}} \backslash \widetilde{\boldsymbol{x}}_{\boldsymbol{i}}\left(\boldsymbol{p}+\boldsymbol{\delta}^{E}, \boldsymbol{\Delta}\right)\right) \leq 1
$$

By Proposition 2,

$$
\underline{\boldsymbol{x}}_{i}, \widetilde{\boldsymbol{x}}_{i}\left(\boldsymbol{p}+\boldsymbol{\delta}^{E}, \boldsymbol{\Delta}\right) \in Q_{i}(\boldsymbol{p})
$$

Hence,

$$
U_{i}\left(\underline{\boldsymbol{x}}_{\boldsymbol{i}}\right)-\boldsymbol{p} \cdot \underline{\boldsymbol{x}}_{\boldsymbol{i}}=U_{i}\left(\widetilde{\boldsymbol{x}}_{\boldsymbol{i}}\left(\boldsymbol{p}+\boldsymbol{\delta}^{E}, \boldsymbol{\Delta}\right)\right)-\boldsymbol{p} \cdot \widetilde{\boldsymbol{x}}_{\boldsymbol{i}}\left(\boldsymbol{p}+\boldsymbol{\delta}^{E}, \boldsymbol{\Delta}\right),
$$

and by equation 24 ,

$$
\left(\widetilde{\boldsymbol{x}}_{\boldsymbol{i}}\left(\boldsymbol{p}+\boldsymbol{\delta}^{E}, \boldsymbol{\Delta}\right)-\underline{\boldsymbol{x}}_{\boldsymbol{i}}\right)\left(\boldsymbol{\delta}^{E}+\underline{\lambda} \boldsymbol{\Delta}\right)=0 .
$$

Then, either

$$
\underline{\lambda} \Delta^{k}=0 \text { for some } k \in K,
$$


a contradiction to Lemma 5 for prices $\boldsymbol{p}+\boldsymbol{\delta}^{E}$ and $\boldsymbol{p}+\boldsymbol{\delta}^{E}+\underline{\lambda} \boldsymbol{\Delta}$, or

$$
\underline{\lambda} \Delta^{k}-\delta=0 \text { for some } k \in K,
$$

a contradiction to equation 23 as $\lambda \boldsymbol{\Delta} \leq \boldsymbol{\delta}^{K}$. An analogous argument can be made for $\widetilde{\boldsymbol{y}}_{\boldsymbol{i}}\left(\boldsymbol{p}-\boldsymbol{\delta}^{E}, \boldsymbol{\Delta}\right)$.

The next proposition shows that for each price vector there exists a unique set of goods which determine the direction in which prices increase (decrease) in the extended algorithm.

A minimal minimizer $\boldsymbol{p}_{+}(\cdot)$ is such that for each $\boldsymbol{p} \in \mathbb{R}_{+}^{K}$,

$$
\boldsymbol{p}_{+}(\boldsymbol{p}) \in \underset{\widetilde{\boldsymbol{p}} \in\left\{\boldsymbol{p}+\boldsymbol{\Delta}: \mathbf{0} \leq \boldsymbol{\Delta} \leq \boldsymbol{\delta}(\boldsymbol{p})^{K}\right\}}{\arg \min }\{L(\widetilde{\boldsymbol{p}})\},
$$

such that for each $\boldsymbol{p}^{\prime} \in \mathbb{R}_{+}^{K}$ if $\boldsymbol{p} \leq \boldsymbol{p}^{\prime} \lesseqgtr \boldsymbol{p}_{+}(\boldsymbol{p})$, then $L\left(\boldsymbol{p}^{\prime}\right)>L\left(\boldsymbol{p}_{+}(\boldsymbol{p})\right)$.

So, a minimal minimizer $\boldsymbol{p}_{+}(\boldsymbol{p})$ is a minimizer of the Lyapunov function of equation 5 in $\left\{\boldsymbol{p}+\boldsymbol{\Delta}: \mathbf{0} \leq \boldsymbol{\Delta} \leq \boldsymbol{\delta}(\boldsymbol{p})^{K}\right\}$ and this set does not contain any price vector which is less than or equal to $\boldsymbol{p}_{+}(\boldsymbol{p})$ in every coordinate, and a minimizer of the Lyapunov function in that set.

Similarly, for each $\boldsymbol{p} \in \mathbb{R}_{+}^{K}$, a maximal minimizer $\boldsymbol{p}_{-}(\cdot)$ is defined as

$$
\boldsymbol{p}_{-}(\boldsymbol{p}) \in \underset{\widetilde{\boldsymbol{p}} \in\left\{\boldsymbol{p}-\boldsymbol{\Delta}: \mathbf{0} \leq \boldsymbol{\Delta} \leq \boldsymbol{\delta}(\boldsymbol{p})^{K}\right\}}{\arg \min }\{L(\widetilde{\boldsymbol{p}})\},
$$

such that for each $\boldsymbol{p}^{\prime} \in \mathbb{R}_{+}^{K}$ if $\boldsymbol{p} \geq \boldsymbol{p}^{\prime} \gtrless \boldsymbol{p}_{-}(\boldsymbol{p})$, then $L\left(\boldsymbol{p}^{\prime}\right)>L\left(\boldsymbol{p}_{-}(\boldsymbol{p})\right)$.

Proposition 3. Suppose that Assumptions A1 - A4 hold, and that bidders truthfully report their demand. Then, at each price $\boldsymbol{p} \in \mathbb{R}_{+}^{K}$, and at each $\delta \in$ $[0, \delta(\boldsymbol{p})]$, there exist a unique minimal minimizer $\boldsymbol{p}_{+}(\boldsymbol{p})$, a unique maximal minimizer $\boldsymbol{p}_{-}(\boldsymbol{p})$, and sets of goods $E_{+}(\boldsymbol{p}), E_{-}(\boldsymbol{p}) \subset K$ such that

$$
\boldsymbol{p}_{+}(\boldsymbol{p})=\boldsymbol{p}+\boldsymbol{\delta}^{E_{+}(\boldsymbol{p})}
$$

and

$$
\boldsymbol{p}_{-}(\boldsymbol{p})=\boldsymbol{p}-\boldsymbol{\delta}^{E_{-}(\boldsymbol{p})} .
$$

Corollary. Suppose that Assumptions A1-A4 hold, and that bidders truthfully report their demand. Then, for each step $t$ of the extended ascending (descending) algorithm, if the procedure does not terminate at $t$, then there exists a step $t^{\prime}>t$ such that there is no step $\hat{t}$ such that $\hat{t} \in\left(t, t^{\prime}\right)$. 
Proof of the Corollary. Let $t \in[0, \infty)$ be a step of the algorithm, and $\boldsymbol{p}(t)$ be the corresponding price vector. If $t$ is not the last step, then, by Propositions 2 and 3, there exist $\delta(\boldsymbol{p})>0$ and $E \subset K$ such that no bidder adds any bundle at any price reached between $\boldsymbol{p}(t)$ and $\boldsymbol{p}(\tilde{t})=\boldsymbol{p}(t)+\boldsymbol{\delta}^{E}$. Hence, there is no step between $t$ and $\tilde{t}$. Let $t^{\prime}=\inf _{\substack{t^{\prime \prime}>t \text { and } \\ t^{\prime \prime} \text { is a step }}} t^{\prime \prime}$. Observe that $t^{\prime}>\tilde{t}$, and $t^{\prime}$ is a step.

Proof. As $L(\cdot)$ is continuous and the set $\left\{\boldsymbol{p}+\boldsymbol{\Delta}: \mathbf{0} \leq \boldsymbol{\Delta} \leq \boldsymbol{\delta}^{K}\right\}$ is compact, $L(\cdot)$ attains a minimum. Thus, by Lemma $2, L(\cdot)$ is a submodular function. Hence, the set of minimizers of $L(\cdot)$ in $\left\{\boldsymbol{p}+\boldsymbol{\Delta}: \mathbf{0} \leq \boldsymbol{\Delta} \leq \boldsymbol{\delta}^{K}\right\}$ are a nonempty sublattice. Therefore, there exists a unique minimal minimizer $\boldsymbol{p}_{+}(\boldsymbol{p})$ of $L(\cdot)$ in $\left\{\boldsymbol{p}+\boldsymbol{\Delta}: \boldsymbol{0} \leq \boldsymbol{\Delta} \leq \boldsymbol{\delta}^{K}\right\}$.

Now, suppose on the contrary that there does not exist $E_{+}(\boldsymbol{p})$ satisfying equation 26. Then, there exists $\bar{k} \in K$ such that $p_{+}^{\bar{k}}(\boldsymbol{p}) \notin\left\{p^{\bar{k}}, p^{\bar{k}}+\delta\right\}$. Let $\widetilde{\boldsymbol{p}}$ be a price vector such that for each $k \in K$

$$
\widetilde{p}^{k}= \begin{cases}p^{k} & \text { if } p_{+}^{k}(\boldsymbol{p}) \notin\left\{p^{k}, p^{k}+\delta\right\} \\ p_{+}^{k}(\boldsymbol{p}) & \text { if } p_{+}^{k}(\boldsymbol{p}) \in\left\{p^{k}, p^{k}+\delta\right\} .\end{cases}
$$

Construct

$$
\widetilde{\Delta}=p_{+}(p)-\widetilde{p},
$$

and

$$
\bar{\lambda}=\frac{\delta}{\max \left\{\widetilde{\Delta}^{k}: k \in K\right\}} .
$$

Observe that as $p_{+}^{\bar{k}}(\boldsymbol{p}) \notin\left\{p^{\bar{k}}, p^{\bar{k}}+\delta\right\}, \widetilde{\boldsymbol{\Delta}} \geqslant \mathbf{0}$, and hence, $\bar{\lambda}$ is well-defined. Moreover, $\boldsymbol{p}_{+}(\boldsymbol{p})$ is a convex combination of $\widetilde{\boldsymbol{p}}+\bar{\lambda} \widetilde{\boldsymbol{\Delta}}$ and $\widetilde{\boldsymbol{p}}$. Note that $\widetilde{\boldsymbol{p}}+$ $\bar{\lambda} \widetilde{\boldsymbol{\Delta}} \leq \boldsymbol{\delta}^{K}$. Hence, Lemma 6 for $E \subset K$ such that $\widetilde{\boldsymbol{p}}=\boldsymbol{p}+\boldsymbol{\delta}^{E}$, and for $\boldsymbol{\Delta}=\widetilde{\boldsymbol{\Delta}}$ implies that for each $\lambda \in[0, \bar{\lambda}]$

$$
\widetilde{\boldsymbol{x}}_{\boldsymbol{i}}(\widetilde{\boldsymbol{p}}, \widetilde{\boldsymbol{\Delta}}) \in Q_{i}(\widetilde{\boldsymbol{p}}+\lambda \widetilde{\boldsymbol{\Delta}}) .
$$

Therefore, for each $\lambda \in[0, \bar{\lambda}] L(\widetilde{\boldsymbol{p}}+\lambda \widetilde{\boldsymbol{\Delta}})$ is linear in $\lambda$.

If $L(\widetilde{\boldsymbol{p}}+\bar{\lambda} \widetilde{\boldsymbol{\Delta}}) \geq L(\widetilde{\boldsymbol{p}})$, then $L(\widetilde{\boldsymbol{p}}) \leq L\left(\boldsymbol{p}_{+}(\boldsymbol{p})\right)$, a contradiction to $\boldsymbol{p}_{+}(\boldsymbol{p})$ being the unique a minimal minimizer. On the other hand, if $L(\widetilde{\boldsymbol{p}}+\bar{\lambda} \widetilde{\boldsymbol{\Delta}})<L(\widetilde{\boldsymbol{p}})$, then $L(\widetilde{\boldsymbol{p}}+\bar{\lambda} \widetilde{\boldsymbol{\Delta}})<L\left(\boldsymbol{p}_{+}(\boldsymbol{p})\right)$, a contradiction to $\boldsymbol{p}_{+}(\boldsymbol{p})$ being a minimizer. So, there exists $E_{+}(\boldsymbol{p})$ satisfying equation 26 .

An analogous argument can be made for $\boldsymbol{p}_{-}(\boldsymbol{p})$ and $E_{-}(\boldsymbol{p})$. 
The goods in $E_{+}(\boldsymbol{p})$ are called the goods in excess demand at price $\boldsymbol{p}$. This notion of excess demand is different from the classical notion of excess demand. Gul and Stacchetti (2000) discuss the need for a different notion of excess demand because of the problems arising during the price adjustment when goods are discrete. Gul and Stacchetti (2000) point out to the distinction between the level of excess demand and the sum of quantities of goods in excess demand in discrete goods. For example, two different goods can be in excess demand but the level of excess demand may only be 1 . The set of goods in excess demand according to the classical notion and the one here coincide if the demand correspondences are assumed to be single-valued. In other words, the same set of goods will be in excess demand.

The following Lemma gives a simple method for determining the set of goods in excess demand.

Lemma 7. Suppose that Assumptions A1-A4 hold, and that bidders truthfully report their demand. Then, for each $\boldsymbol{p} \in \mathbb{R}_{+}^{K}$

$$
E_{+}(\boldsymbol{p}) \in \underset{E \subset K}{\arg \min } \sum_{k \in E}\left(S_{k}-\sum_{i \in N} \widetilde{x}_{i}^{k}\left(\boldsymbol{p}, \mathbf{1}^{E}\right)\right),
$$

and for each $\delta \in[0, \delta(\boldsymbol{p}))$

$$
E_{+}\left(\boldsymbol{p}+\boldsymbol{\delta}^{E_{+}(\boldsymbol{p})}\right)=E_{+}(\boldsymbol{p}) .
$$

Moreover, if $t$ and $t^{\prime}$ are two consecutive steps of the extended ascending algorithm such that $t<t^{\prime}$, then for all $\hat{t} \in\left[t, t^{\prime}\right)$

$$
E_{+}(\boldsymbol{p}(\hat{t}))=E_{+}(\boldsymbol{p}(t)) .
$$

Similarly,

$$
E_{-}(\boldsymbol{p}) \in \underset{E \subset K}{\arg \max } \sum_{k \in E}\left(S_{k}-\sum_{i \in N} \widetilde{y}_{i}^{k}\left(\boldsymbol{p}, \mathbf{1}^{E}\right)\right)
$$

and for each $\delta \in[0, \delta(\boldsymbol{p}))$,

$$
E_{-}\left(\boldsymbol{p}-\boldsymbol{\delta}^{E_{-}(\boldsymbol{p})}\right)=E_{-}(\boldsymbol{p}) .
$$

Moreover, if $t$ and $t^{\prime}$ are two consecutive steps of the extended descending algorithm such that $t<t^{\prime}$, then for all $\hat{t} \in\left[t, t^{\prime}\right)$

$$
E_{-}(\boldsymbol{p}(\hat{t}))=E_{-}(\boldsymbol{p}(t)) .
$$


Proof. For each $\boldsymbol{p} \in \mathbb{R}_{+}^{K}$, by Proposition 3 , there exist $\delta(\boldsymbol{p}) \in \mathbb{R}_{++}$and $E_{+}(\boldsymbol{p}) \subset K$ such that $\boldsymbol{p}_{+}(\boldsymbol{p})=\boldsymbol{p}+\boldsymbol{\delta}(\boldsymbol{p})^{E_{+}(\boldsymbol{p})}$. As

$$
\boldsymbol{\delta}(\boldsymbol{p})^{E_{+}(\boldsymbol{p})} \cdot \boldsymbol{x}=\delta(\boldsymbol{p}) \sum_{k \in E_{+}(\boldsymbol{p})} x^{k}
$$

by Proposition 2 and by the definition of $\widetilde{\boldsymbol{x}}_{\boldsymbol{i}}\left(\boldsymbol{p}, \mathbf{1}^{E_{+}(\boldsymbol{p})}\right)$, for each $i \in N$, and for each $\delta \in(0, \delta(\boldsymbol{p})]$

$$
\widetilde{\boldsymbol{x}}_{\boldsymbol{i}}\left(\boldsymbol{p}, \mathbf{1}^{E_{+}(\boldsymbol{p})}\right) \in Q_{i}\left(\boldsymbol{p}+\boldsymbol{\delta}^{E_{+}(\boldsymbol{p})}\right) .
$$

Observe that

$$
\begin{aligned}
L\left(\boldsymbol{p}+\boldsymbol{\delta}^{E_{+}(\boldsymbol{p})}\right)= & \left(\boldsymbol{p}+\boldsymbol{\delta}^{E_{+}(\boldsymbol{p})}\right) \cdot \boldsymbol{S} \\
& \left.+\sum_{i \in N}\left(U_{i}\left(\widetilde{\boldsymbol{x}}_{\boldsymbol{i}}\left(\boldsymbol{p}, \mathbf{1}^{E_{+}(\boldsymbol{p})}\right)\right)-\left(\boldsymbol{p}+\boldsymbol{\delta}^{E_{+}(\boldsymbol{p})}\right) \cdot \widetilde{\boldsymbol{x}}_{\boldsymbol{i}}\left(\boldsymbol{p}, \mathbf{1}^{E_{+}(\boldsymbol{p})}\right)\right)\right) \\
= & \boldsymbol{p} \cdot \boldsymbol{S}+\sum_{i \in N}\left(U_{i}\left(\widetilde{\boldsymbol{x}}_{\boldsymbol{i}}\left(\boldsymbol{p}, \mathbf{1}^{E_{+}(\boldsymbol{p})}\right)-\boldsymbol{p} \cdot \widetilde{\boldsymbol{x}}_{\boldsymbol{i}}\left(\boldsymbol{p}, \mathbf{1}^{E_{+}(\boldsymbol{p})}\right)\right)+\boldsymbol{\delta}^{E_{+}(\boldsymbol{p})} \cdot \boldsymbol{S}\right. \\
& \left.-\sum_{i \in N} \boldsymbol{\delta}^{E_{+}(\boldsymbol{p})} \cdot \widetilde{\boldsymbol{x}}_{\boldsymbol{i}}\left(\boldsymbol{p}, \mathbf{1}^{E_{+}(\boldsymbol{p})}\right)\right) \\
= & \left.L(\boldsymbol{p})+\boldsymbol{\delta}^{E_{+}(\boldsymbol{p})} \cdot \boldsymbol{S}-\sum_{i \in N} \boldsymbol{\delta}^{E_{+}(\boldsymbol{p})} \cdot \widetilde{\boldsymbol{x}}_{\boldsymbol{i}}\left(\boldsymbol{p}, \mathbf{1}^{E_{+}(\boldsymbol{p})}\right)\right) .
\end{aligned}
$$

So, minimizing $L\left(\boldsymbol{p}+\boldsymbol{\delta}^{E}\right)$, and maximizing $\left.L(\boldsymbol{p})-L\left(\boldsymbol{p}+\boldsymbol{\delta}^{E}\right)\right)$ over the set of goods $E \subset K$ is equivalent to

$$
\min _{E \subset K} \delta \sum_{k \in E}\left(S_{k}-\sum_{i \in N} \widetilde{x}_{i}^{k}\left(\boldsymbol{p}, \mathbf{1}^{E}\right)\right) .
$$

Hence, for each $\delta, \delta^{\prime} \in(0, \delta(\boldsymbol{p})]$, the set of solutions to equation 29 for $\delta$ and $\delta^{\prime}$ are identical.

Thus,

$$
E_{+}(\boldsymbol{p}) \in \underset{E \subset K}{\arg \min } \sum_{k \in E}\left(S_{k}-\sum_{i \in N} \widetilde{x}_{i}^{k}\left(\boldsymbol{p}, \mathbf{1}^{E}\right)\right),
$$

and for each $\delta \in(0, \delta(\boldsymbol{p})]$

$$
E_{+}\left(\boldsymbol{p}+\boldsymbol{\delta}^{E_{+}(\boldsymbol{p})}\right)=E_{+}(\boldsymbol{p}) .
$$


An analogous derivation can be made for $E_{-}(\boldsymbol{p})$. Observe that at each equilibrium price vector $\boldsymbol{p}^{*}$,

$$
E_{+}\left(\boldsymbol{p}^{*}\right)=E_{-}\left(\boldsymbol{p}^{*}\right)=\emptyset .
$$

Note that Lemma 7 implies that in the extended ascending algorithm and the extended descending algorithm, bidders reporting their demand sets at the initial price vector and at prices at which they add a bundle to their demand sets gives sufficient information to the auctioneer to adjust prices correctly.

\subsection{Reaching Walrasian Equilibrium Prices in Finite Steps}

In this section, I will show that the extended algorithms terminate in any economy as long as Assumptions $A 1-A 4$ are satisfied. Moreover, the extended ascending (descending) algorithm reaches a Walrasian equilibrium price vector in finitely many steps if initial prices are sufficiently low (high).

Proposition 4 shows how the price vector at which the extended algorithm terminates relates to the set of Walrasian equilibrium price vectors.

Proposition 4. Suppose that Assumptions A1 - A4 hold, and that bidders truthfully report their demand. Starting from any initial price vector $\boldsymbol{p}(0) \in \mathbb{R}_{+}^{K}$, if the extended ascending algorithm ends at $T$, then $\boldsymbol{p}(T) \geq \underline{\boldsymbol{p}}$. Similarly, starting from any initial price $\boldsymbol{p}(0) \in \mathbb{R}_{+}^{K}$, if the extended descending algorithm ends at $T$, then $\boldsymbol{p}(T) \leq \overline{\boldsymbol{p}}$.

Proof. Suppose, for the extended ascending algorithm, that $\boldsymbol{p}(T) \nsupseteq \underline{\boldsymbol{p}}$. Then, there exists a good $k \in K$ such that the price of good $k$ at $\boldsymbol{p}(T) \wedge \underline{\boldsymbol{p}}$ is less than $\underline{p}^{k}$. Since $\underline{\boldsymbol{p}}$ is the smallest Walrasian equilibrium price vector, $\overline{\boldsymbol{p}}(T) \wedge \underline{\boldsymbol{p}}$ is not a Walrasian equilibrium price vector. Therefore, by Proposition 1

$$
L(\underline{\boldsymbol{p}})<L(\boldsymbol{p}(T) \wedge \underline{\boldsymbol{p}}) .
$$

As $L(\cdot)$ is submodular by Lemma 2 ,

$$
L(\boldsymbol{p}(T) \wedge \underline{\boldsymbol{p}})+L(\boldsymbol{p}(T) \vee \underline{\boldsymbol{p}}) \leq L(\boldsymbol{p}(T))+L(\underline{\boldsymbol{p}}) .
$$


Inequalities 30 and 31 imply that

$$
L(\boldsymbol{p}(T) \vee \underline{\boldsymbol{p}})<L(\boldsymbol{p}(T)) .
$$

By Propositions 2 and 3, and by the definition of $\delta(\cdot)$ in equation 10 , there exist $\delta(\boldsymbol{p}(T)) \in \mathbb{R}_{++}$and $E(\boldsymbol{p}(T)) \subset K$ such that $\boldsymbol{p}(T)+\boldsymbol{\delta}(\boldsymbol{p})^{E(\boldsymbol{p}(T))}$ is the unique minimal minimizer of $L(\cdot)$ in the cube $\left\{\boldsymbol{p}+\boldsymbol{\Delta}: \mathbf{0} \leq \boldsymbol{\Delta} \leq \boldsymbol{\delta}(\boldsymbol{p}(T))^{K}\right\}$. Let $\boldsymbol{p}^{\prime}$ be a strict convex combination of $\boldsymbol{p}(T)$ and $\boldsymbol{p}(T) \vee \boldsymbol{p}$ such that $\mathbf{0} \leq$ $\boldsymbol{p}^{\prime}-\boldsymbol{p}(T) \leq \boldsymbol{\delta}(\boldsymbol{p}(T))^{K} . \boldsymbol{p}^{\prime}$ exists as $\boldsymbol{p}(T) \vee \underline{\boldsymbol{p}} \geqslant \boldsymbol{p}(T)$. Convexity of $L(\cdot)$ implies that $L\left(\boldsymbol{p}^{\prime}\right)<L(\boldsymbol{p}(T))$. This means that $E(\boldsymbol{p}(T)) \neq \emptyset$ and that $\boldsymbol{p}(T)$ is not a minimizer in $\left\{\boldsymbol{p}(T)+\boldsymbol{\Delta}: \mathbf{0} \leq \boldsymbol{\Delta} \leq \boldsymbol{\delta}(\boldsymbol{p}(T))^{K}\right\}$. Therefore, the extended ascending algorithm does not stop at $T$ as it is possible to decrease the Lyapunov function further, a contradiction. Hence, $\boldsymbol{p}(T) \geq \underline{\boldsymbol{p}}$.

The result for the extended descending algorithm can be proven analogously.

Proposition 5 shows that if the initial prices are smaller (larger) than the lowest (highest) Walrasian equilibrium price vector, then all the price vectors reached by the extended ascending (descending) algorithm are smaller (larger) than the lowest (highest) Walrasian equilibrium price vector.

Proposition 5. Suppose that Assumptions A1 - A4 hold, and that bidders truthfully report their demand. In the extended ascending algorithm, starting from any initial price vector $\boldsymbol{p}(0) \in \mathbb{R}_{+}^{K}$, if $\boldsymbol{p}(t) \leq \underline{\boldsymbol{p}}$, then $\boldsymbol{p}\left(t^{\prime}\right) \leq \underline{\boldsymbol{p}}$ for all $t^{\prime}>t$. In the extended descending algorithm, starting from any initial price vector $\boldsymbol{p}(0) \in \mathbb{R}_{+}^{K}$, if $\boldsymbol{p}(t) \geq \overline{\boldsymbol{p}}$, then $\boldsymbol{p}\left(t^{\prime}\right) \geq \overline{\boldsymbol{p}}$ for all $t^{\prime}>t$.

Proof. Consider the extended ascending algorithm. Suppose, on the contrary, that there exist $t$ and $t^{\prime}$ such that $t^{\prime}>t, \boldsymbol{p}(t) \leq \boldsymbol{p}$, and $p\left(t^{\prime}\right)^{k}>p^{k}$ for some $k \in K$. As $\boldsymbol{p}(\cdot)$ is continuous, there exists $t^{\prime \prime}$ such that $t<\overline{t^{\prime \prime}}<t^{\prime}$ and $p\left(t^{\prime \prime}\right)^{k}=\underline{p}^{k}$. Let

$$
\overline{t^{\prime \prime}}=\sup _{\boldsymbol{p}(t) \leq \underline{p}} t .
$$

Since $\boldsymbol{p}(\cdot)$ is continuous, and prices are ascending, $\overline{t^{\prime \prime}}$ exists and $p\left(\overline{t^{\prime \prime}}\right)^{k}=p^{k}$. By Proposition 1, and as $\underline{\boldsymbol{p}}$ is a Walrasian price vector, for each $s>\overline{t^{\prime \prime}}$,

$$
L(\underline{\boldsymbol{p}}) \leq L(\boldsymbol{p}(s) \vee \underline{\boldsymbol{p}}) .
$$

Moreover, since $L(\cdot)$ is a submodular function by Lemma 2,

$$
L(\boldsymbol{p}(s) \vee \underline{\boldsymbol{p}})+L(\boldsymbol{p}(s) \wedge \underline{\boldsymbol{p}}) \leq L(\boldsymbol{p}(s))+L(\underline{\boldsymbol{p}}) .
$$


Inequalities 32 and 33 imply that

$$
L(\boldsymbol{p}(s) \wedge \underline{\boldsymbol{p}}) \leq L(\boldsymbol{p}(s)) .
$$

Note that there exists $k^{\prime} \in K$ such that for each $s>\overline{t^{\prime \prime}}$

$$
p^{k^{\prime}}(s) \wedge \underline{p}^{k^{\prime}}<p^{k^{\prime}}(s) .
$$

By Proposition 3, there exists $E \subset K$ such that

$$
\boldsymbol{p}(\bar{s})=\boldsymbol{p}\left(\overline{t^{\prime \prime}}\right)+\boldsymbol{\delta}\left(\boldsymbol{p}\left(\overline{t^{\prime \prime}}\right)\right)^{E} .
$$

for some $\bar{s} \in\left(\overline{t^{\prime \prime}}, t^{\prime}\right)$. Since inequality 35 holds for $s=\bar{s}$,

$$
\boldsymbol{p}(\bar{s}) \wedge \underline{\boldsymbol{p}}=\boldsymbol{p}\left(\overline{t^{\prime \prime}}\right)+\boldsymbol{\delta}\left(\boldsymbol{p}\left(\overline{t^{\prime \prime}}\right)\right)^{A}
$$

for some $A \subsetneq E$. Therefore, by inequality $34, \boldsymbol{p}(\bar{s})$ is not a minimal minimizer, a contradiction.

The result for the extended descending algorithm can be proven analogously.

The next step (Theorem 1) is to show that the extended ascending algorithm and the extended descending algorithm stop after finitely many steps.

Theorem 1. Suppose that Assumptions A1-A4 hold, and that bidders truthfully report their demands. Then, the extended ascending algorithm and the extended descending algorithm terminate in finite steps.

Proof. The algorithm converges to a price vector. Consider the extended ascending algorithm. As there are finite number of bidders and the consumption set of each bidder is bounded, there is an upper bound on bidders' values for each good. In other words, for each good $k \in K$, there is a price $p_{\text {max }}^{k} \in \mathbb{R}_{+}$at and above which no bidder wants good $k$ regardless of the prices of the rest of the goods . Observe that, by Lemma 7, if the total quantity demanded of a good $k \in K$ at price vector $\boldsymbol{p}(t) \in \mathbb{R}_{+}^{K}$ is less than the total quantity of good $k$ available, then the price of good $k$ will not be in the set of prices that will be increased at $t$ in the extended ascending algorithm. Therefore, in the extended ascending algorithm, for each $k \in K$ and for each $t \in[0, \infty)$,

$$
p^{k}(t) \leq p_{\max }^{k}
$$


By the Corollary to Proposition 3, for each step $t^{\prime}$, there exists a step $t>t^{\prime}$ such that there is no other step $\tilde{t}$ such that $t^{\prime}<\tilde{t}<t$, and there exists a rational number $t_{q} \in \mathbb{Q}$ such that $t^{\prime}<t_{q}<t$. Therefore, there are at most countably many steps in the extended ascending algorithm. Let $\left\{\boldsymbol{p}_{s}\right\}_{s \in \sigma}$ be the sequence of all price vectors reached by the extended ascending algorithm at all steps.

Observe that, by construction of the extended ascending algorithm, if $t^{\prime}>t^{\prime \prime}$, then $\boldsymbol{p}\left(t^{\prime}\right) \gtrless \boldsymbol{p}\left(t^{\prime \prime}\right)$. Therefore, for each $s \in \sigma$,

$$
p_{s} \lesseqgtr p_{s+1}
$$

As the sequence $\left\{\boldsymbol{p}_{\boldsymbol{s}}\right\}_{s \in \sigma}$ is bounded above and monotonically increasing, it converges to a price vector $\boldsymbol{p}^{*} \in \mathbb{R}_{+}$such that $\boldsymbol{p}^{*} \leq\left(p_{\max }^{k}\right)_{k \in K}$.

The relationship between the demand sets at price vectors of consecutive steps. Note that for each $s \in \sigma, \boldsymbol{p}_{\boldsymbol{s}+\mathbf{1}}$ is the first price vector where a bidder adds a bundle to his demand set after $\boldsymbol{p}_{\boldsymbol{s}}$ in the extended ascending algorithm. Since the set of prices that are increased does not change between consecutive steps in the extended ascending algorithm, the same set of prices are increased from $\boldsymbol{p}_{\boldsymbol{s}}$ to $\boldsymbol{p}_{\boldsymbol{s}+\mathbf{1}}$. Therefore, there exist $\delta_{s}>0$ and $E_{s} \subset K$ such that

$$
\boldsymbol{p}_{\boldsymbol{s}+\mathbf{1}}=\boldsymbol{p}_{\boldsymbol{s}}+\boldsymbol{\delta}_{\boldsymbol{s}}^{E_{s}},
$$

which can be rewritten as

$$
\boldsymbol{p}_{\boldsymbol{s}+\mathbf{1}}=\boldsymbol{p}_{\mathbf{0}}+\sum_{s^{\prime}=0}^{s} \boldsymbol{\delta}_{\boldsymbol{s}^{\prime}} E_{s^{\prime}}
$$

where $\delta_{s^{\prime}}>0$ and $E_{s^{\prime}} \subset K$ for each $s^{\prime} \in \mathbb{Z}$ such that $0 \leq s^{\prime} \leq s$.

For each step $s \in \sigma$, and for each bidder $i_{s} \in N$ who adds a bundle to his demand set at price vector $\boldsymbol{p}_{\boldsymbol{s}+\mathbf{1}}$, by Lemma 5 for price vectors $\boldsymbol{p}_{\boldsymbol{s}}$ and $\boldsymbol{p}_{\boldsymbol{s + 1}}$, there exist bundles

$$
\boldsymbol{x}_{\boldsymbol{i}_{\boldsymbol{s}}} \in Q_{i}\left(\boldsymbol{p}_{\boldsymbol{s}}\right) \text { and } \boldsymbol{x}_{\boldsymbol{i}_{\boldsymbol{s}}}^{\prime} \in Q_{i}\left(\boldsymbol{p}_{\boldsymbol{s}+\mathbf{1}}\right) \backslash Q_{i}\left(\boldsymbol{p}_{\boldsymbol{s}}\right)
$$

such that

$$
\#\left(\boldsymbol{x}_{\boldsymbol{i}_{\boldsymbol{s}}} \backslash \boldsymbol{x}_{\boldsymbol{i}_{\boldsymbol{s}}}^{\prime}\right) \leq 1 \text { and } \#\left(\boldsymbol{x}_{\boldsymbol{i}_{\boldsymbol{s}}}^{\prime} \backslash \boldsymbol{x}_{\boldsymbol{i}_{\boldsymbol{s}}}\right) \leq 1 \text {, }
$$

and there exists a unique $k \in K$ such that $k \in E_{s}$ and

$$
x_{i_{s}}^{k}=x_{i_{s}}^{\prime k}+1
$$


and there exists at most one $k^{\prime} \in K$ such that $k^{\prime} \in K \backslash E_{s}$ and

$$
x_{i_{s}}^{k^{\prime}}=x_{i_{s}}^{\prime k^{\prime}}-1 .
$$

The strict monotonicity of excess demand. For each $s \in \sigma$, let $t_{s} \in[0, \infty)$ be such that

$$
\boldsymbol{p}_{s}=\boldsymbol{p}\left(t_{s}\right) \text {. }
$$

Now I will show that the total quantity of goods in excess demand strictly decreases at each step, i.e.

$$
\sum_{\hat{k} \in E_{s+1}}\left(S_{\hat{k}}-\sum_{i \in N} \widetilde{x}_{i}^{\hat{k}}\left(\boldsymbol{p}_{\boldsymbol{s}+\mathbf{1}}, \mathbf{1}_{s+1}^{E}\right)\right)>\sum_{\hat{k} \in E_{s}}\left(S_{\hat{k}}-\sum_{i \in N} \widetilde{x}_{i}^{\hat{k}}\left(\boldsymbol{p}_{\boldsymbol{s}}, \mathbf{1}_{s}^{E}\right)\right) .
$$

As the prices in $E_{s}$ are increased from $\boldsymbol{p}_{\boldsymbol{s}}$ to $\boldsymbol{p}_{\boldsymbol{s}+\mathbf{1}}$, by Lemma 3, for each $i \in N$ and for all $t, t^{\prime} \in\left(t_{s}, t_{s+1}\right)$

$$
Q_{i}(\boldsymbol{p}(t))=Q_{i}\left(\boldsymbol{p}\left(t^{\prime}\right)\right)
$$

and for each $t \in\left[t_{s}, t_{s+1}\right)$

$$
Q_{i}\left(\boldsymbol{p}_{\boldsymbol{s}}\right) \supset Q_{i}(\boldsymbol{p}(t))
$$

and by the definition of step,

$$
Q_{i}(\boldsymbol{p}(t)) \subset Q_{i}\left(\boldsymbol{p}_{\boldsymbol{s}+\mathbf{1}}\right) .
$$

Therefore, for each $t \in\left(t_{s}, t_{s+1}\right)$

$$
Q_{i}(\boldsymbol{p}(t)) \subset Q_{i}\left(\boldsymbol{p}_{\boldsymbol{s}}\right) \cap Q_{i}\left(\boldsymbol{p}_{\boldsymbol{s}+\mathbf{1}}\right),
$$

and by Lemma 3

$$
Q_{i}(\boldsymbol{p}(t))=Q_{i}\left(\boldsymbol{p}_{\boldsymbol{s}}\right) \cap Q_{i}\left(\boldsymbol{p}_{\boldsymbol{s}+\mathbf{1}}\right) .
$$

For each $i \in N$ and for each $t \in\left(t_{s}, t_{s+1}\right)$, by equation 40 , and by the definition of $\widetilde{\boldsymbol{x}}_{i}(\cdot)$,

$$
\sum_{\hat{k} \in E_{s+1}} \widetilde{x}_{i}^{\hat{k}}\left(\boldsymbol{p}_{\boldsymbol{s + 1}}, \mathbf{1}_{s+1}^{E}\right) \leq \sum_{\hat{k} \in E_{s+1}} \widetilde{x}_{i}^{\hat{k}}\left(\boldsymbol{p}(t), \mathbf{1}_{s+1}^{E}\right) .
$$

Therefore, for each $t \in\left(t_{s}, t_{s+1}\right)$

$$
\sum_{\hat{k} \in E_{s+1}}\left(S_{\hat{k}}-\sum_{i \in N} \widetilde{x}_{i}^{\hat{k}}\left(\boldsymbol{p}_{\boldsymbol{s + 1}}, \mathbf{1}_{s+1}^{E}\right)\right) \geq \sum_{\hat{k} \in E_{s+1}}\left(S_{\hat{k}}-\sum_{i \in N} \widetilde{x}_{i}^{\hat{k}}\left(\boldsymbol{p}(t), \mathbf{1}_{s+1}^{E}\right)\right) .
$$


Since, by Lemma 7 , for each $t \in\left(t_{s}, t_{s+1}\right)$

$$
\sum_{\hat{k} \in E_{s+1}}\left(S_{\hat{k}}-\sum_{i \in N} \widetilde{x}_{i}^{\hat{k}}\left(\boldsymbol{p}(t), \mathbf{1}_{s+1}^{E}\right)\right) \geq \sum_{\hat{k} \in E_{s}}\left(S_{\hat{k}}-\sum_{i \in N} \widetilde{x}_{i}^{\hat{k}}\left(\boldsymbol{p}(t), \mathbf{1}_{s}^{E}\right)\right)
$$

and

$$
\sum_{\hat{k} \in E_{s}}\left(S_{\hat{k}}-\sum_{i \in N} \widetilde{x}_{i}^{\hat{k}}\left(\boldsymbol{p}(t), \mathbf{1}_{s}^{E}\right)\right)=\sum_{\hat{k} \in E_{s}}\left(S_{\hat{k}}-\sum_{i \in N} \widetilde{x}_{i}^{\hat{k}}\left(\boldsymbol{p}_{\boldsymbol{s}}, \mathbf{1}_{s}^{E}\right),\right.
$$

the following inequality holds

$$
\sum_{\hat{k} \in E_{s+1}}\left(S_{\hat{k}}-\sum_{i \in N} \widetilde{x}_{i}^{\hat{k}}\left(\boldsymbol{p}_{\boldsymbol{s}+\mathbf{1}}, \mathbf{1}_{s+1}^{E}\right)\right) \geq \sum_{\hat{k} \in E_{s}}\left(S_{\hat{k}}-\sum_{i \in N} \widetilde{x}_{i}^{\hat{k}}\left(\boldsymbol{p}_{\boldsymbol{s}}, \mathbf{1}_{s}^{E}\right)\right) .
$$

Now I will show that the inequality 42 is strict.

Claim. There exists a bidder $i_{s}^{\prime} \in N$ such that there does not exist $\tilde{\boldsymbol{x}}_{\boldsymbol{i}_{\boldsymbol{s}}^{\prime}} \in$ $Q_{i_{s}^{\prime}}\left(\boldsymbol{p}_{\boldsymbol{s}}\right) \cap Q_{i_{s}^{\prime}}\left(\boldsymbol{p}_{\boldsymbol{s}+\mathbf{1}}\right)$ satisfying equation 8 at $\left(\boldsymbol{p}_{\boldsymbol{s}+\mathbf{1}}, \mathbf{1}_{s+1}^{E}\right)$.

Proof of the Claim. Suppose, on the contrary, that this is not true. Then, for each $i \in N$ there exists $\tilde{\boldsymbol{x}}_{\boldsymbol{i}} \in Q_{i}\left(\boldsymbol{p}_{\boldsymbol{s}}\right) \cap Q_{i}\left(\boldsymbol{p}_{\boldsymbol{s}+\mathbf{1}}\right)$ satisfying equation 8 at $\left(\boldsymbol{p}_{s+\mathbf{1}}, \mathbf{1}_{s+1}^{E}\right)$. Then,

$$
\sum_{\hat{k} \in E_{s+1}}\left(S_{\hat{k}}-\sum_{i \in N} \widetilde{x}_{i}^{\hat{k}}\left(\boldsymbol{p}_{\boldsymbol{s}+\mathbf{1}}, \mathbf{1}_{s+1}^{E}\right)\right)=\sum_{\hat{k} \in E_{s}}\left(S_{\hat{k}}-\sum_{i \in N} \tilde{x}_{i}^{\hat{k}}\right) .
$$

As

$$
\sum_{\hat{k} \in E_{s}}\left(S_{\hat{k}}-\sum_{i \in N} \widetilde{x}_{i}^{\hat{k}}\left(\boldsymbol{p}_{\boldsymbol{s}}, \mathbf{1}_{s}^{E}\right)\right)=\sum_{\hat{k} \in E_{s}}\left(S_{\hat{k}}-\sum_{i \in N} \tilde{x}_{i}^{\hat{k}}\right),
$$

by inequality $42, E_{s}=E_{s+1}$. But equation 38 implies that for each bidder $i_{s}$ who adds a bundle at step $t_{s+1}$

$$
\sum_{\hat{k} \in E_{s}} \tilde{x}_{i_{s}}^{\hat{k}}>\sum_{\hat{k} \in E_{s}} x_{i_{s}}^{\prime \hat{k}}
$$

because

$$
\sum_{\hat{k} \in E_{s}} x_{i_{s}}^{\hat{k}}>\sum_{\hat{k} \in E_{s}} x_{i_{s}}^{\hat{k}^{k}}
$$


and

$$
\sum_{\hat{k} \in E_{s}} \tilde{x}_{i_{s}}^{\hat{k}}=\sum_{\hat{k} \in E_{s}} x_{i_{s}}^{\hat{k}},
$$

where $\boldsymbol{x}_{\boldsymbol{i}_{\boldsymbol{s}}}^{\prime}$ and $\boldsymbol{x}_{\boldsymbol{i}_{\boldsymbol{s}}}$ are bundles satisfying equations 36 and inequalities 37. However, $E_{s}=E_{s+1}$ and inequality 43 imply that $\tilde{\boldsymbol{x}}_{\boldsymbol{i}_{\boldsymbol{s}}} \in Q_{i_{s}}\left(\boldsymbol{p}_{\boldsymbol{s}}\right) \cap Q_{i_{s}}\left(\boldsymbol{p}_{\boldsymbol{s}+\mathbf{1}}\right)$ does not satisfy equation 8 at $\left(\boldsymbol{p}_{\boldsymbol{s + 1}}, \mathbf{1}_{s+1}^{E}\right)$, a contradiction.

Therefore, there exists a bidder $i_{s}^{\prime} \in N$ such that there does not exist $\tilde{\boldsymbol{x}}_{\boldsymbol{i}_{\boldsymbol{s}}^{\prime}} \in Q_{i_{s}^{\prime}}\left(\boldsymbol{p}_{\boldsymbol{s}}\right) \cap Q_{i_{s}^{\prime}}\left(\boldsymbol{p}_{\boldsymbol{s}+\mathbf{1}}\right)$ satisfying equation 8 at $\left(\boldsymbol{p}_{\boldsymbol{s}+\mathbf{1}}, \mathbf{1}_{s+1}^{E}\right)$. So, bidder $i_{s}^{\prime}$ adds a bundle to his demand set at $\boldsymbol{p}_{\boldsymbol{s}+\mathbf{1}}$, and there exists $\boldsymbol{x}_{\boldsymbol{i}_{\boldsymbol{s}}^{\prime}}^{\prime} \in Q_{i_{s}^{\prime}}\left(\boldsymbol{p}_{\boldsymbol{s}+\mathbf{1}}\right)$ satisfying equation 8 at $\left(\boldsymbol{p}_{\boldsymbol{s + 1}}, \mathbf{1}_{s+1}^{E}\right)$.

Hence,

$$
\sum_{\hat{k} \in E_{s+1}} x_{i_{s}^{\prime}}^{\prime \hat{k}}<\sum_{\hat{k} \in E_{s+1}} \tilde{x}_{i_{s}^{\prime}}^{\hat{k}}
$$

for all $\tilde{\boldsymbol{x}}_{\boldsymbol{i}_{s}^{\prime}} \in Q_{i^{\prime}}\left(\boldsymbol{p}_{\boldsymbol{s}}\right) \cap Q_{i^{\prime}}\left(\boldsymbol{p}_{\boldsymbol{s}+\mathbf{1}}\right)$.

Inequality 44 implies that inequality 41 is strict for $i_{s}^{\prime}$. So, inequality 42 is strict.

The algorithm has finitely many steps. As the left side of inequality 39 is integer and since the extended ascending algorithm terminates whenever it is positive, there are finitely many steps.

Observe that the definition of step implies that if there is no market clearing allocation at a step, then there will not be a market clearing allocation until the next step. Therefore, the extended ascending algorithm terminates at price vector $\boldsymbol{p}_{|\boldsymbol{\sigma}|-\mathbf{1}}=\boldsymbol{p}(T)=\boldsymbol{p}^{*} \in \mathbb{R}_{+}^{K}$ for some finite $T$.

The proof for the extended descending algorithm can be done analogously.

Theorem 2 (Theorem 3) shows that if the initial prices are sufficiently low (high), then the extended ascending (descending) algorithm reaches the lowest (highest) Walrasian equilibrium price vector of the economy in finitely many steps.

Theorem 2. Suppose that Assumptions A1-A4 hold, and that bidders truthfully report their demands. Then, starting from any initial price vector of $\boldsymbol{p}(0) \in \mathbb{R}_{+}^{K}$ such that $\boldsymbol{p}(0) \leq \boldsymbol{p}$, the extended ascending algorithm reaches the lowest Walrasian equilibrium price vector $\underline{\boldsymbol{p}}$ in finite steps. 
Proof. The auctioneer asks each bidder $i \in N$ his demand set $x_{i}(\boldsymbol{p}(0)) \subset$ $X_{i}$ at $\boldsymbol{p}(0)$. Using these demand sets, the auctioneer determines the set $E_{+}(\boldsymbol{p}(0)) \subset K$ of goods in excess demand at $\boldsymbol{p}(0)$ (see Lemma 7 ). Prices of these goods in $E_{+}(\boldsymbol{p}(0))$ are increased continuously at the same rate while the rest remains the same. As prices are increased, at any time $t \in[0, \infty)$, if there is a bidder who adds a bundle to his demand set, then the price adjustment stops. Each bidder $i$ reports his demand $x_{i}(\boldsymbol{p}(t)) \subset X_{i}$ at $\boldsymbol{p}(t)$. The auctioneer determines the set $E_{+}(\boldsymbol{p}(t)) \subset K$ of goods in excess demand at $\boldsymbol{p}(t)$ (see Lemma 7 ). Prices of goods in $E_{+}(\boldsymbol{p}(t))$ are increased continuously at the same rate while the rest remains the same. The extended ascending algorithm reaches some price vector in finite steps (see Theorem 1). By Propositions 4 and 5, the price vector the extended ascending algorithm reaches is $\boldsymbol{p}$, and by Proposition 1, there exists a market clearing allocation $\left(\boldsymbol{x}_{\boldsymbol{i}}^{*}\right)_{i \in N}$ such that $\boldsymbol{x}_{\boldsymbol{i}}^{*} \in x_{i}(\underline{\boldsymbol{p}})$ for each $i \in N$, provided that bidders report their demands truthfully.

The proof of the convergence of the extended descending algorithm to the highest Walrasian equilibrium price, Theorem 3 below, is analogous to the proof of Theorem 2.

Theorem 3. Suppose that Assumptions A1-A4 hold, and that bidders truthfully report their demands. Then, starting from any initial price vector of $\boldsymbol{p}(0) \in \mathbb{R}_{+}^{K}$ such that $\boldsymbol{p}(0) \geq \overline{\boldsymbol{p}}$, the extended descending algorithm reaches the highest Walrasian equilibrium price vector $\overline{\boldsymbol{p}}$ in finite steps.

A price vector $\boldsymbol{p}^{\prime} \in \mathbb{R}_{+}^{K}$ is a supporting price vector if there exists an allocation $\boldsymbol{x}^{\prime}=\left(\boldsymbol{x}_{\boldsymbol{i}}^{\prime}\right)_{i \in N}$ such that $\sum_{i \in N} \boldsymbol{x}_{\boldsymbol{i}} \leq \boldsymbol{S}$, and for each $i \in N, \boldsymbol{x}_{\boldsymbol{i}}^{\prime} \in$ $Q_{i}\left(\boldsymbol{p}^{\prime}\right)$.

Theorem 2 imposes restrictions on the initial price vector. Theorem 4 shows that if these conditions are violated, then the extended ascending algorithm reaches in finite steps a supporting price vector whenever bidders report their demand sets truthfully.

Theorem 4. Suppose that Assumptions A1-A4 hold, and that bidders truthfully report their demand. Let $\boldsymbol{p}(T)$ be a price vector the extended ascending algorithm reaches at $T \in[0, \infty)$. Then, the extended ascending algorithm ends at $\boldsymbol{p}(T)$ in finite steps if and only if $\boldsymbol{p}(T)$ is a supporting price vector.

Proof. Define a new economy by adding a fictitious bidder 0 with the consumption set $X_{0}=\left\{\boldsymbol{x}_{\mathbf{0}} \in \mathbb{Z}^{K}: \mathbf{0} \leq \boldsymbol{x}_{\mathbf{0}} \leq \boldsymbol{S}\right\}$, and utility function $U_{0}\left(\boldsymbol{x}_{\mathbf{0}}\right)=$ 
$\boldsymbol{p}(T) \cdot \boldsymbol{x}_{\mathbf{0}}$ to the economy. So, the indirect utility function of bidder 0 is $V_{0}(\boldsymbol{p})=((\boldsymbol{p}(T)-\boldsymbol{p}) \vee \mathbf{0}) \cdot \boldsymbol{S}$. Observe that $\boldsymbol{p}(T)$ is a Walrasian equilibrium price of the economy with bidder 0 if and only if $\boldsymbol{p}(T)$ is a supporting price vector of the economy without bidder 0 . The Lyapunov function for the economy with bidder 0 is $\widetilde{L}(\boldsymbol{p})=\boldsymbol{p} \cdot \boldsymbol{S}+\sum_{i=0}^{n} V_{i}(\boldsymbol{p})$, and

$$
\begin{aligned}
\widetilde{L}(\boldsymbol{p}(T)) & =\boldsymbol{p}(T) \cdot \boldsymbol{S}+\sum_{i=0}^{n} V_{i}(\boldsymbol{p}(T)) \\
& =\boldsymbol{p}(T) \cdot \boldsymbol{S}+\sum_{i=1}^{n} V_{i}(\boldsymbol{p}(T)) .
\end{aligned}
$$

Since $V_{i}(\boldsymbol{p}) \geq V_{i}(\boldsymbol{p}(T) \vee \boldsymbol{p})$ for any $i \in N \cup\{0\}, \widetilde{L}(\boldsymbol{p}) \geq \widetilde{L}(\boldsymbol{p}(T) \vee \boldsymbol{p})$ for any $\boldsymbol{p} \in \mathbb{R}_{++}^{K}$. Now suppose that the price adjustment procedure in the economy with bidder 0 terminates at $\boldsymbol{p}(T)$. Then, $\boldsymbol{p}(T)$ minimizes $\widetilde{L}(\cdot)$. Suppose on the contrary that there exists $\boldsymbol{p} \in \mathbb{R}_{++}^{K}, \boldsymbol{p} \neq \boldsymbol{p}(T)$, such that $\widetilde{L}(\boldsymbol{p})<\widetilde{L}(\boldsymbol{p}(T))$. Observe that $\widetilde{L}(\boldsymbol{p} \vee \boldsymbol{p}(T))<\widetilde{L}(\boldsymbol{p}(T))$. So, for any $\delta(\boldsymbol{p}) \in \mathbb{R}, \delta(\boldsymbol{p})>0$, there exists $\boldsymbol{p}^{\prime} \in\left\{\boldsymbol{p}(T)+\boldsymbol{\Delta}: \mathbf{0} \lesseqgtr \boldsymbol{\Delta} \leq \boldsymbol{\delta}(\boldsymbol{p})^{K}\right\}$ such that $\boldsymbol{p}^{\prime}$ is a strict convex combination of $\boldsymbol{p} \vee \boldsymbol{p}(T)$ and $\boldsymbol{p}(T)$. As, by Lemma $2, \widetilde{L}(\cdot)$ is a convex function, $\widetilde{L}\left(\boldsymbol{p}^{\prime}\right)<\widetilde{L}(\boldsymbol{p}(T))$. This contradicts to the assumption that the algorithm terminated at $\boldsymbol{p}(T)$.

Since $\boldsymbol{p}(T)$ is a minimizer of $\widetilde{L}(\cdot)$, applying Proposition 1 to $\widetilde{L}(\cdot), \boldsymbol{p}(T)$ is a Walrasian equilibrium price of the economy with bidder 0 . Therefore $\boldsymbol{p}(T)$ is a supporting price of the economy without bidder 0 . By Theorem 1, the price adjustment procedure converges in finite steps.

\subsection{Truthful Bidding is an Ex Post Efficient Equilib- rium}

In the tâtonnement algorithm of Ausubel (2006), prices are adjusted in discrete time and they take integer values. Ausubel (2006) converts the tâtonnement algorithm to a continuous procedure by linearly increasing prices between consecutive integer-valued price vectors, and uses the continuoustime procedure to show that sincere bidding is an efficient equilibrium Ausubel's auction, and the procedure yields Walrasian equilibrium prices. The extended tâtonnement algorithm is also a continuous-time procedure. Observe that the extended tâtonnement algorithm and the continuous version of the 
tâtonnement algorithm follow the same path if bidders' values for bundles are integer, the initial price vector is integer-valued, and the extended ascending algorithm has a unit rate of change.

The price adjustment process can be written as

$$
\frac{\mathrm{d} p^{k}(t)}{\mathrm{d} t}= \begin{cases}c_{E(\boldsymbol{p}(t))} & \text { if } k \in E(\boldsymbol{p}(t)) \\ 0 & \text { if } k \notin E(\boldsymbol{p}(t))\end{cases}
$$

for all $t$ except when $t$ is a step, and

$$
E(\boldsymbol{p}(t))=E_{+}(\boldsymbol{p}(t)) \text { and } c_{E_{+}(\boldsymbol{p}(t))}>0
$$

for the extended ascending algorithm, and

$$
E(\boldsymbol{p}(t))=E_{-}(\boldsymbol{p}(t)) \text { and } c_{E_{-}(\boldsymbol{p}(t))}<0
$$

for the extended descending algorithm.

By Lemma 1 in Ausubel (2006), if $\boldsymbol{p}(\cdot)$ is continuous and for each $j \in$ $N, j \neq i$, any $k \in K x_{j}^{k}(\cdot)$, amount of good $k$ demanded by bidder $j$, is of bounded variation, then $a_{i}(T)$ is well-defined. Moreover, if $\boldsymbol{p}(\cdot)$ is also a piecewise smooth function from $[0, T]$ to $\mathbb{R}^{K}$, and for each $j \in N, j \neq i, U_{j}(\cdot)$ is a concave and continuous function, then by Lemma 2 in Ausubel (2006)

$$
a_{i}(T)=\boldsymbol{p}(0) \cdot\left[\boldsymbol{S}-\boldsymbol{x}_{-\boldsymbol{i}}(0)\right]-\sum_{j \neq i, j \in N}\left[U_{j}\left(\boldsymbol{x}_{\boldsymbol{j}}(\boldsymbol{p}(T))\right)-U_{j}\left(\boldsymbol{x}_{\boldsymbol{j}}(\boldsymbol{p}(0))\right)\right] .
$$

Observe that the price path induced by the extended ascending (descending) algorithm is piecewise linear and continuous. Therefore, since substitutes preferences imply concave utility functions (see Ausubel (2006), p.617), the conditions of Lemmas 1 and 2 in Ausubel (2006) hold. So, by Lemmas 1 and 2 in Ausubel (2006), payment function given in equation 4 is well-defined and path-independent, and the equality 45 holds for the extended tâtonnement algorithms. Hence, Theorems $1^{\prime}$ and $2^{\prime}$ in Ausubel (2006) hold literally (see the discussion on p.620 Ausubel (2006)) for the extension of the Ausubel auction proposed in this paper. As a result, starting at a sufficiently low (high) price vector, the truthful bidding constitutes an efficient equilibrium in the extension of Ausubel's auction, and the extended ascending (descending) algorithm terminates at a Walrasian equilibrium price vector in finitely many steps. 


\section{Conclusion}

In this paper, I extended the ascending and the descending price adjustment procedures proposed in Ausubel (2006) to real-valued quasilinear utility functions. I show that these extended procedures converge to a Walrasian equilibrium price vector in finite steps. Unlike the tâtonnement algorithm in Ausubel (2006), the extended tâtonnement algorithm does not require any information on bidders' when bidders' values for bundles real.

\section{References}

Ausubel, L. M. (2004): "An Efficient Ascending-Bid Auction for Multiple Objects," American Econ. Rev., 94, 1452-1475.

(2005): "Walrasian Tatonnement for Discrete Goods," mimeo.

(2006): "An Efficient Dynamic Auction for Heterogeneous Commodities," American Econ. Rev., 96, 602-629.

Ausubel, L. M., and P. Cramton (2002): "Demand Reduction and Inefficiency in Multi-Unit Auctions," mimeo.

BikhChandani, S., And J. W. Mamer (1997): "Competitive Equilibrium in an Exchange Economy with Indivisibilities," J. Econ. Theory, 74, 385413.

Gul, F., And E. Stacchetti (1999): "Walrasian Equilibrium with Gross Substitutes," J. Econ. Theory, 87, 95-124.

(2000): "The English Auction with Differentiated Commodities," J. Econ. Theory, 92, 66-95.

Krishna, V. (2002): Auction Theory. Academic Press.

Milgrom, P. (2004): Putting Auction Theory to Work. Cambridge University Press.

Milgrom, P., And B. Strulovici (2009): "Substitute Goods, Auctions, and Equilibrium," J. Econ. Theory, 144, 212-247. 
Simon, L. K., And M. B. Stinchcombe (1989): "Extensive Form Games in Continuous Time: Pure Strategies," Econometrica, 57(5), 1171-1214.

Vickrey, W. (1961): "Counterspeculation, Auctions, and Competitive Sealed Tenders," Journal of Finance, 16, 8-37. 